\title{
Interannual variations of early winter Antarctic polar stratospheric cloud formation and nitric acid observed by CALIOP and MLS
}

\author{
Alyn Lambert, Michelle L. Santee, and Nathaniel J. Livesey \\ Jet Propulsion Laboratory, California Institute of Technology, Pasadena, California, USA \\ Correspondence to: Alyn Lambert (alyn.lambert@jpl.nasa.gov)
}

Received: 19 May 2016 - Published in Atmos. Chem. Phys. Discuss.: 13 June 2016

Revised: 17 November 2016 - Accepted: 21 November 2016 - Published: 8 December 2016

\begin{abstract}
We use satellite-borne measurements collected over the last decade (2006-2015) from the Aura Microwave Limb Sounder (MLS) and the Cloud-Aerosol Lidar with Orthogonal Polarization (CALIOP) to investigate the nitric acid distribution and the properties of polar stratospheric clouds (PSCs) in the early winter Antarctic vortex. Frequently, at the very start of the winter, we find that synoptic-scale depletion of $\mathrm{HNO}_{3}$ can be detected in the inner vortex before the first lidar detection of geophysically associated PSCs. The generation of "sub-visible" PSCs can be explained as arising from the development of a solid particle population with low number densities and large particle sizes. Assumed to be composed of nitric acid trihydrate (NAT), the sub-visible PSCs form at ambient temperatures well above the ice frost point, but also above the temperature at which supercooled ternary solution (STS) grows out of the background supercooled binary solution (SBS) distribution. The temperature regime of their formation, inferred from the simultaneous uptake of ambient $\mathrm{HNO}_{3}$ into NAT and their Lagrangian temperature histories, is at a depression of a few kelvin with respect to the NAT existence threshold, $T_{\mathrm{NAT}}$. Therefore, their nucleation requires a considerable supersaturation of $\mathrm{HNO}_{3}$ over NAT, and is consistent with a recently described heterogeneous nucleation process on solid foreign nuclei immersed in liquid aerosol. We make a detailed investigation of the comparative limits of detection of PSCs and the resulting sequestration of $\mathrm{HNO}_{3}$ imposed by lidar, mid-infrared, and microwave techniques. We find that the temperature history of air parcels, in addition to the local ambient temperature, is an important factor in the relative frequency of formation of liquid/solid PSCs. We conclude that the initiation of NAT nucleation and the subsequent development of large NAT particles capable of sedimentation and denitrification in the early winter do
\end{abstract}

not emanate from an ice-seeding process. Finally, we investigate the patterns of interannual variability and compare the relative formation frequency of liquid and solid PSCs in the Antarctic lower polar stratosphere using the results of a cluster analysis to synthesize the combined CALIOP and MLS measurements into a relatively small number of interrelated categories.

\section{Introduction}

In Lambert et al. (2012) we reported on the formation of polar stratospheric clouds (PSCs) and the initial stages of denitrification in the early 2008 Antarctic PSC season. The first appearance of PSCs was observed through the uptake of gas-phase $\mathrm{HNO}_{3}$ by MLS and by patchy lidar backscatter detection by CALIOP. Although the uptake of $\mathrm{HNO}_{3}$ was substantial, the low lidar backscatter and ambient temperatures indicated a nitric acid trihydrate (NAT) composition rather than supercooled ternary solution (STS). An inference of large particle sizes with radii $\geq 5-7 \mu \mathrm{m}$ and low NAT number density $\left(<1 \times 10^{-3} \mathrm{~cm}^{-3}\right)$ was made from the combination of the temperature, $\mathrm{HNO}_{3}$, and backscatter data, further confirmed by the timescale of the appearance of an enhanced $\mathrm{HNO}_{3}$ layer at $68 \mathrm{hPa}$, caused by sedimentation of NAT from above and evaporation back into the gas phase. We also determined that in 2008 a NAT polar freezing belt (Tabazadeh et al., 2001; Höpfner et al., 2006a), generated by gravity-wave induced ice-seeding, occurred after the first appearance of large particle NAT clouds; i.e., the latter were not causally linked to the mountain wave seeding events. In Lambert et al. (2012) and Pitts et al. (2013), we studied the uptake of $\mathrm{HNO}_{3}$ by different types of PSCs classified by CALIOP 
as a function of temperature. We showed that the distributions of gas-phase $\mathrm{HNO}_{3}$ vs. temperature combined with the independent CALIOP PSC classification (Pitts et al., 2009) provide valuable insights into the PSC formation process. Liquid STS particles exhibit well-defined equilibrium properties, whereas the liquid/solid particle STS/NAT mixtures exhibit non-equilibrium properties resulting from kinetically limited growth. In this paper we expand our previous investigations to include the distribution of the lidar backscatter of different PSC types as a function of temperature and $\mathrm{HNO}_{3}$ uptake. We also report on the interannual variability in the Antarctic PSC seasons from 2006 to 2015.

The discrimination between different PSC types at temperatures above the ice frost point, $T_{\mathrm{ICE}}$, stemming either from the growth of STS on the background liquid supercooled binary solution (SBS) or the nucleation of NAT, provides critical observations enabling validation of theoretical PSC formation pathways. In the case of NAT, the nucleation processes are still not understood in detail. Whether homogeneous or heterogeneous nucleation is in force, it is the nucleated NAT number density that provides the key to the subsequent microphysical development of the NAT clouds, since rapid nucleation at high supersaturations leads to higher NAT number densities with small particle radii, whereas slow nucleation at low supersaturations produces low NAT number densities and allows the particles to grow to much larger sizes (Jensen et al., 2002). The homogeneous nucleation of NAT from STS and the production of large-size NAT in the 2010/2011 Arctic winter has been simulated in the SDWACCM/CARMA PSC model (Whole-Atmosphere Community Climate Model with Specified Dynamics with the Community Aerosol and Radiation Model for Atmospheres) as described by Zhu et al. (2015). In this model, homogeneous nucleation rates were determined using the nucleation equations derived from laboratory experiments by Tabazadeh et al. (2002), with the free energy tuned by less than $10 \%$. The same nucleation rates were found by Zhu et al. (2016) to reproduce the observed timing of PSC formation during the Antarctic winter of 2011. In contrast, Hoyle et al. (2013) used an extension of the Zurich Optical and Microphysical box Model (ZOMM) (Luo et al., 2003) to include a new pathway of heterogeneous formation of ice and NAT on solid foreign nuclei inclusions, originating from meteoritic dust, that are assumed to be present in at least $50 \%$ of all aerosol drops (Curtius et al., 2005).

Hoyle et al. (2013) determined that NAT can form heterogeneously at some considerable vapor supersaturation, at temperatures well above the ice frost point, on the solid foreign nuclei immersed in STS. Only a limited number of surface inhomogeneities on the foreign nuclei provide favorable active sites such that the NAT nucleation barrier is depressed sufficiently for nucleation to occur. Once the most efficient active sites have caused nucleation at a particular supersaturation, the remaining population of STS/foreign particles have lower quality active sites that require either a higher supersaturation to increase the nucleation rate or waiting for a longer period of time for nucleation at the same supersaturation to occur. Three tuning parameters are used to control the heterogeneous nucleation rate in the ZOMM model: nucleation barrier, nucleation strength, and compatibility factor. These were adjusted with consideration of the detection thresholds applied to the model results (Engel et al., 2013; Hoyle et al., 2013), to replicate successfully the CALIOP backscatter observations for a representative orbit. The tuned model was then used to facilitate intercomparisons with the CALIOP observations during December 2009 and to verify the model results for a few case studies in the Arctic (Engel et al., 2013; Hoyle et al., 2013). However, the low visibility of the NAT PSCs, as we indicated in Lambert et al. (2012), poses a detection challenge for lidar backscatter techniques, even though the accompanying $\mathrm{HNO}_{3}$ sequestration in NAT can be substantial and detectable by other instruments as a decrease in the gas-phase $\mathrm{HNO}_{3}$.

In situ measurements of NAT particles on a synoptic scale, with a large particle mode of around $15 \mu \mathrm{m}$ diameter, from the Forward Scattering Spectrometer Probe (FSSP) (Molleker et al., 2014), and observations of their attendant denitrification (Woiwode et al., 2014), were reported during the Arctic RECONCILE (reconciliation of essential process parameters for an enhanced predictability of Arctic stratospheric ozone) campaign (von Hobe et al., 2013). In order to resolve discrepancies involving implied condensed $\mathrm{HNO}_{3}$ above that available from the gas phase (including additional $\mathrm{HNO}_{3}$ brought down by renitrification) and growth/sedimentation rates that are not commensurate with back-trajectories, those authors hypothesized that the particles are non-compact or highly aspherical or NAT-coated ice. Grooß et al. (2014) coupled a saturation-dependent parameterization of the ZOMM heterogeneous NAT scheme with the Chemical Lagrangian Model of the Stratosphere (CLaMS) and determined that derived PSC properties were in better agreement with CALIOP data than for simulations using a constant rate NAT nucleation, thus confirming the results of Hoyle et al. (2013). Furthermore, Grooß et al. (2014) compared their simulations to the Arctic in situ aerosol size distributions from FSSP in January 2010 and found that the observed large NAT particles (exceeding $15 \mu \mathrm{m}$ in diameter) were not present in the co-located simulations. However, the CLaMS simulations did develop particle distributions with median NAT diameters of up to $20 \mu \mathrm{m}$ at other times in the 2009/2010 Arctic winter. Hence, the new ZOMM nucleation scheme is quite capable of producing large-size NAT, and Grooß et al. speculate that the presence of a highly nonspherical NAT component coupled with the orientation of the particles within the FSSP sample volume may lead to an overestimation of their actual size. Zhu et al. (2015) also found that particles with diameters of $20 \mu \mathrm{m}$ were produced with their homogeneous nucleation scheme, which does not require that there are special nuclei only available under certain conditions. 
PSC formation processes at play in the Arctic are clearly applicable to the Antarctic, and the recent observations from RECONCILE add support to the conclusions of our previous work (Lambert et al., 2012), which highlighted the appearance of synoptic-scale large particle NAT in the early Antarctic winter of 2008. Obviously, the hypotheses concerning the origin and microphysical characteristics of these socalled "NAT rocks" will be a challenge to validate without further in situ observations. However, the decade-long record of overlapping spaceborne CALIOP and MLS measurements presents an opportunity to develop improved algorithms for the extraction of information on PSCs and to apply newfound knowledge to the understanding of their current and future role in ozone depletion.

In Sect. 2 we review the satellite instruments and atmospheric measurements used in our analyses. The temperature history of an air parcel and the relation to heterogeneous nucleation of NAT is explored. We introduce a compact alternative visualization to the standard graphical representation of satellite orbit plots that enables easier comprehension of several parameters plotted at multiple atmospheric levels and spanning many days of observations. In Sect. 3 we investigate the limits of detection of equilibrium STS and STS/NAT mixtures separately for lidar backscatter, mid-infrared extinction, and uptake of $\mathrm{HNO}_{3}$ from the gas phase. We also investigate STS and NAT PSCs in terms of the distributions in a three-parameter space of $\mathrm{HNO}_{3}$, backscatter, and temperature. We compare these with the CALIOP PSC classification scheme, which uses fixed regions within a two-parameter discrimination domain (depolarization vs. total backscatter). In Sect. 4 we show orbit transects and time series of co-located CALIOP and MLS data. These are used to investigate the relative formation of liquid/solid PSCs and the resulting denitrification and renitrification. In Sect. 5 the early stages of formation of Antarctic PSCs at 68-21 hPa in 2009 are examined using CALIOP PSC types and Lagrangian temperature history, with the inference of an initial population of subvisible solid-particle NAT clouds superseded by a predominantly liquid STS composition over a period of about a week. Finally, the interannual variability of the early Antarctic PSC seasons from 2006 to 2015 is discussed in Sect. 6.

\section{Datasets and methodology}

The Cloud-Aerosol Lidar with Orthogonal Polarization (CALIOP) dual-wavelength elastic backscatter lidar (Winker et al., 2009) flies on the Cloud-Aerosol Lidar and Infrared Pathfinder Satellite Observations (CALIPSO) satellite launched in April 2006. The Microwave Limb Sounder (MLS) is onboard the Aura spacecraft launched in July 2004. CALIPSO and Aura are part of the NASA/ESA afternoon "A-train" satellite constellation at $705 \mathrm{~km}$ nominal altitude and $98^{\circ}$ inclination, with daily global coverage attained in 14.5 orbits. The initial A-train configuration of the CALIPSO and Aura spacecraft from April 2006 to April 2008 resulted in an across-track orbit offset of $\sim 200 \mathrm{~km}$, with the MLS tangent point leading the CALIOP nadir view by about $7.5 \mathrm{~min}$. Since April 2008 Aura and CALIPSO have been operated to maintain positioning within tightly constrained control boxes, such that the MLS tangent point and the CALIOP nadir view are co-located to better than about $10-20 \mathrm{~km}$ and about $30 \mathrm{~s}$.

We derive co-located meteorological data from the Goddard Earth Observing System Data Assimilation System (GEOS-5 DAS). The 6-hourly synoptic gridded data products (Rienecker et al., 2008) of temperatures and winds are supplied on a 540 by 361 longitude-latitude grid. The GEOS5 data are interpolated in location and time to the MLS alongtrack data. Parcel temperature histories are obtained from the MLS Lagrangian Trajectory Diagnostic (LTD) dataset (Livesey et al., 2015), which consists of 15-day forward and reverse trajectories launched from a curtain of points along the Aura MLS observation track. The advection calculations are based on Manney et al. (1994), with wind fields and diabatic heating rates taken from the 3-hourly ModernEra Retrospective Analysis for Research and Applications (MERRA-2) dataset (Bosilovich et al., 2015). Advances in both the GEOS-5 model and the assimilation system, including GPS Radio Occultation datasets, are included in MERRA-2. The integration uses a fourth-order Runge-Kutta scheme with a $5 \mathrm{~min}$ time step, and saved trajectory locations and temperatures are output every $30 \mathrm{~min}$. The MLS derived meteorological products (DMPs) (Manney et al., 2007) are used where necessary to identify measurement locations that lie within the Antarctic vortex based on the potential vorticity field, sPV $<-1.4$, scaled in "vorticity units" (Manney et al., 1994).

\subsection{CALIOP PSC data}

We use the CALIOP Level-1b v3 standard data product to extract information on PSCs (as documented in Lambert et al., 2012) at a $50 \mathrm{~km}$ horizontal by $0.5 \mathrm{~km}$ vertical resolution. We also use a recently released Level- 2 operational dataset L2PSCMask (v1 Polar Stratospheric Cloud Mask Product) produced by the CALIPSO science team. The Level-2 operational data consist of nighttime-only data and contain profiles of PSC presence, composition, optical properties, and meteorological information along the CALIPSO orbit tracks at $5 \mathrm{~km}$ horizontal by $180 \mathrm{~m}$ vertical resolution.

We have determined that the v1 L2PSCMask operational product has an incorrect separation of the MIX1 and MIX2 classifications and does not follow the boundary specification given by Pitts et al. (2009) since it failed to include in MIXI those observations with a total scattering ratio of less than 1.25. Separation of PSCs into the MIXI and MIX2 classes is somewhat arbitrary, but provides useful information on the NAT number densities (Pitts et al., 2009). 
The following three-step algorithm (using the Scientific Data Set variable names supplied with the CALIPSO Hierarchical Data Files (HDF) files) has been applied to generate the correct MIX1 and MIX2 classes:

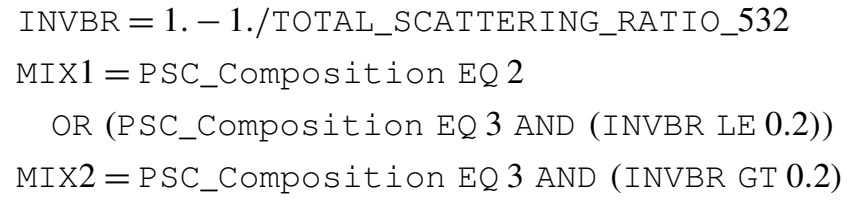

\subsection{MLS gas-phase constituents}

The Microwave Limb Sounder measures thermal emission at millimeter and sub-millimeter wavelengths from the Earth's limb (Waters et al., 2006) along the forward direction of the Aura spacecraft flight track, with a vertical scan from the surface to $90 \mathrm{~km}$ every $24.7 \mathrm{~s}$. Each orbit consists of 240 scans spaced at $1.5^{\circ}(165 \mathrm{~km})$ along-track, with a total of almost 3500 profiles per day and a latitudinal coverage of $82^{\circ} \mathrm{S}$ to $82^{\circ} \mathrm{N}$. The Level-1 limb radiance measurements are inverted using 2-D optimal estimation (Livesey et al., 2006) to produce Level-2 profiles of atmospheric temperature and composition. Validation of the MLS $\mathrm{H}_{2} \mathrm{O}$ and $\mathrm{HNO}_{3}$ data products and error estimations are discussed in detail by Read et al. (2007), Lambert et al. (2007), and Santee et al. (2007). Here we use the MLS version 4 (v4) data (Livesey et al., 2016) with single-profile precisions (accuracies) of 4-15\% (4-7\%) for $\mathrm{H}_{2} \mathrm{O}$ and $0.6 \mathrm{ppbv}$ (1-2 ppbv) for $\mathrm{HNO}_{3}$.

\subsection{Temperature history and relation to NAT nucleation and growth processes}

In this work we frequently apply a convenient temperature coordinate transformation, $T-T_{\text {ICE }}$, by using MLS $\mathrm{H}_{2} \mathrm{O}$ to calculate the ice frost point, in order to remove height-related variations due to changes in the $\mathrm{H}_{2} \mathrm{O}$ partial pressure (Lambert et al., 2012; Pitts et al., 2013). As in Lambert et al. (2012) we quantify the duration of exposure of an air parcel to low temperatures by defining the temperature threshold exposure (TTE) as the total integrated time the air parcel is subject to synoptic-scale temperatures below the chosen threshold. We use a threshold of $T_{\mathrm{ICE}}+4 \mathrm{~K}$ (approximately $T_{\mathrm{NAT}}-3.5 \mathrm{~K}$ ) to demonstrate empirically the correlation of TTE with the uptake of $\mathrm{HNO}_{3}$ by NAT PSCs. The temperature history follows a diabatic back-trajectory for up to 15 days obtained from the MLS LTD dataset (Livesey et al., 2015). The TTE is the total time (in days) that an air parcel has been exposed to temperatures below $T_{\mathrm{ICE}}+4 \mathrm{~K}$ since the last time the temperature fell below $T_{\mathrm{NAT}}$ and remained below $T_{\mathrm{NAT}}$ consistently; i.e., any number of episodes of cooling below $T_{\mathrm{ICE}}+4 \mathrm{~K}$ are accumulated provided that the air parcel has remained consistently below $T_{\mathrm{NAT}}$. The $\mathrm{HNO}_{3}$ and $\mathrm{H}_{2} \mathrm{O}$ values (for estimating $T_{\mathrm{NAT}}$ and $T_{\mathrm{ICE}}$ ) are assumed to be constant from the start point of the back-trajectories. For typical lower stratospheric polar conditions $\left(5 \mathrm{ppmv} \mathrm{H}_{2} \mathrm{O}\right.$,
$10 \mathrm{ppbv} \mathrm{HNO}_{3}$, and $46 \mathrm{hPa}$ ), values for $T_{\mathrm{ICE}}$ and $T_{\mathrm{NAT}}$ are 188 and $195 \mathrm{~K}$, respectively. Both $T_{\mathrm{ICE}}$ and $T_{\mathrm{NAT}}$ are lowered (raised) by about $2 \mathrm{~K}$ at $32 \mathrm{hPa}(68 \mathrm{hPa})$. Under denitrified conditions ( $5 \mathrm{ppbv} \mathrm{HNO}_{3}$ ), $T_{\mathrm{NAT}}$ is lowered by about $1 \mathrm{~K}$, and under dehydrated conditions ( 3 ppmv $\left.\mathrm{H}_{2} \mathrm{O}\right), T_{\text {ICE }}$ is lowered by about $3 \mathrm{~K}$. In denitrified and dehydrated conditions, $T_{\mathrm{NAT}}$ is also lowered by about $3 \mathrm{~K}$. TTE is a remarkably good indicator of the geographical extent of the $\mathrm{HNO}_{3}$ depletion in the vortex (Lambert et al., 2012). Here, we explore the correspondence of TTE to NAT nucleation and growth processes.

According to the development of NAT along a sample trajectory shown by Hoyle et al. (2013) in their Fig. 1, substantial nucleation begins only for temperatures below $T_{\mathrm{NAT}}-4 \mathrm{~K}$. We investigate the temperature-time domain of the nucleation process in Fig. 1, for both (a) early season unperturbed and (b) late season denitrified atmospheres, and calculate the resulting NAT number densities using the heterogeneous nucleation scheme given in Hoyle et al. (2013). The figure serves to illustrate the general properties of NAT cloud formation, but in reality the temperature-time path is important for the modeling of specific clouds. The nucleation rate is a strong function of temperature, and the nucleated NAT shows an almost step-like transition over a narrow temperature range. In contrast, the variation of NAT density with exposure time is more gradual because the nucleation rate at a fixed temperature (i.e., fixed supersaturation) depends only on the integration over time. Exposure to temperatures of $T_{\mathrm{NAT}}-2.2 \mathrm{~K}$ and above produces negligible NAT densities $\left(<10^{-6} \mathrm{~cm}^{-3}\right)$, even for time durations exceeding a month, whereas exposures of $\sim 1$ day to temperatures near $T_{\mathrm{NAT}}-4 \mathrm{~K}$ produce NAT densities 3 orders of magnitude greater $\left(\sim 10^{-3} \mathrm{~cm}^{-3}\right)$. At lower temperatures, the NAT saturation ratio is limited by uptake of $\mathrm{HNO}_{3}$ from the gas phase by STS (Luo et al., 2003), causing the curvature of the NAT density contours below $T_{\mathrm{NAT}}-4.5 \mathrm{~K}$. Denitrification has little effect on the sharp temperature transition, but at lower temperatures where STS forms there is a visible decrease in the NAT number density generated for the same time duration. At nucleation, the NAT particle sizes are small and no larger than the progenitor aerosol particle (SBS or STS). Hence, considerable growth of the NAT particles is required before they can be detected using remote sensing techniques. TTE can be viewed as a proxy for the time elapsed since nucleation occurred, i.e., as a measure of the effective growth time of the NAT particles. The NAT volume density increases gradually through sequestration of $\mathrm{HNO}_{3}$ from the ambient gas phase, and the particles may not achieve their much larger equilibrium size until several days following nucleation.

\subsection{Visual representation of satellite orbital data}

Figure 2a shows typical orbit tracks of the MLS $\mathrm{HNO}_{3}$ distribution mapped onto a polar stereographic projection for ascending and descending orbits at $32 \mathrm{hPa}$. Each col- 

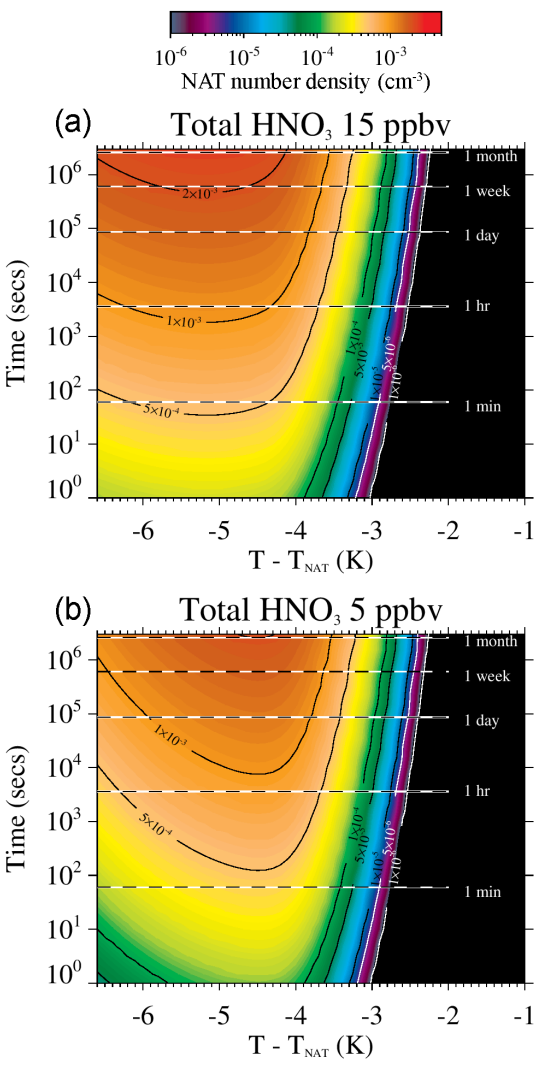

Figure 1. (a) Calculated NAT number densities (colored shading and labeled contour lines) resulting from varying temperature exposure durations in an unperturbed atmosphere with $15 \mathrm{ppbv}$ total $\mathrm{HNO}_{3}$. Horizontal dashed lines highlight exposures of between 1 min and 1 month. An air parcel (containing the requisite background aerosol embedded with foreign nuclei) exposed to a temperature of $T-T_{\mathrm{NAT}}=-4 \mathrm{~K}$ for 1 day will generate $0.0012 \mathrm{~cm}^{-3}$ of nucleated NAT particles. (b) As for (a) except for a denitrified atmosphere with 5 ppbv total $\mathrm{HNO}_{3}$.

ored square is centered at the corresponding MLS profile latitude-longitude retrieval location. The along-track spacing is $1.5^{\circ}$ (distance between centers of the squares). Note that the dimensions of the squares are not related to the MLS along-track (several hundred kilometers for $\mathrm{HNO}_{3}$ ) or across-track (about $10 \mathrm{~km})$ resolutions. Orbit numbering $(0$ is the day of start orbit) is shown around the $60^{\circ} \mathrm{S}$ latitude circle. The color scheme indicates contrasting colors either side of 10 ppbv. Over the 10-day period (26 May to 5 June 2009, Fig. 2a and b), the $\mathrm{HNO}_{3}$ values decrease and the area with values $<10 \mathrm{ppbv}$ is seen to increase and move eastward. Such "satellite data orbit plots" are commonly used, but they do not scale up easily for viewing multiple quantities and pressure levels over periods of the order of a month; e.g., tracking the evolution of four parameters over 20 days at four distinct atmospheric levels requires the digestion of $320 \mathrm{im}-$ ages.
We present an alternative scheme, designed to improve the data visualization, with some similarities to the familiar Hovmöller diagram, but with the abscissa following selected sections of the satellite orbit track rather than running along a zonal or meridional circle. Figure $2 \mathrm{c}$ shows the same data as in Fig. 2a, but replotted as a time-ordered sequence of the along-track points. The MLS orbit tracks are unfolded along the abscissa as a function of the along-track angle $\left(1.5^{\circ}\right.$ is the angular spacing), where the along-track angle of $30^{\circ}$ corresponds to the closest approach of the orbit to the South Pole (at about latitude $82^{\circ} \mathrm{S}$ ). Also shown for convenience are the along-track distances (in kilometers) and the corresponding latitudes and solar zenith angles. The MLS measurement time (hours since start of day at 00:00 UT) is on the ordinate. The orbit numbers are given next to the righthand ordinate. Again, the dimensions of the squares are not related to the MLS orbital track spacing or the MLS measurement time (each complete vertical atmospheric profile is accumulated over about $25 \mathrm{~s}$ ). The main purpose of this compact visual representation (i.e., a sparse "raster" image) is that it enables the "raw" daily observations to be stacked into a longer time series without involving gridding onto a map projection. Note that there is a geographical data void (within $8^{\circ}$ of the pole; see Fig. 2a and b) that is not apparent in the raster representation. MLS looks forward in the alongtrack direction, so it never actually looks into the $8^{\circ}$ polar cap; nor does CALIOP with its nadir view. While the low $\mathrm{HNO}_{3}$ in the vortex rotates eastward in the orbit track plot, this motion translates into a time displacement in the raster plot. The ascending/descending tracks do not intersect in the raster plot and the terminator is always on the rightmost side (a feature that could be potentially useful for examining diurnal species such as $\mathrm{ClO}$ ).

\section{Detection and classification of PSCs}

Improvements in modeling capabilities drive a commensurate need for a thorough evaluation of the observational aspects of PSC research, such as biases in temperature analyses, derived air parcel temperature histories along trajectories and instrument measurement biases and uncertainties. This is highlighted in a specific example shown by Hoyle et al. (2013) in the Arctic on 26 December 2009 (their Fig. 7, orbit 26_04), where they indicate that the ZOMM model predicts a secondary area of NAT clouds between longitudes 12 and $53^{\circ}$, whereas the CALIOP observations do not show a corresponding lidar detection. Hoyle et al. (2013) noted the prolonged exposure time $(80 \mathrm{~h})$ resulting in sedimentation of NAT as a potential cause of the lack of a coincident lidar signal from CALIOP in the L2PSCMask product. However, we have determined that smoothing of the CALIOP L2PSCMask data using a $5 \times 5$ median filter to improve signal-to-noise does in fact indicate the presence of PSCs. In Fig. 3, we compare the along-track cross sections 

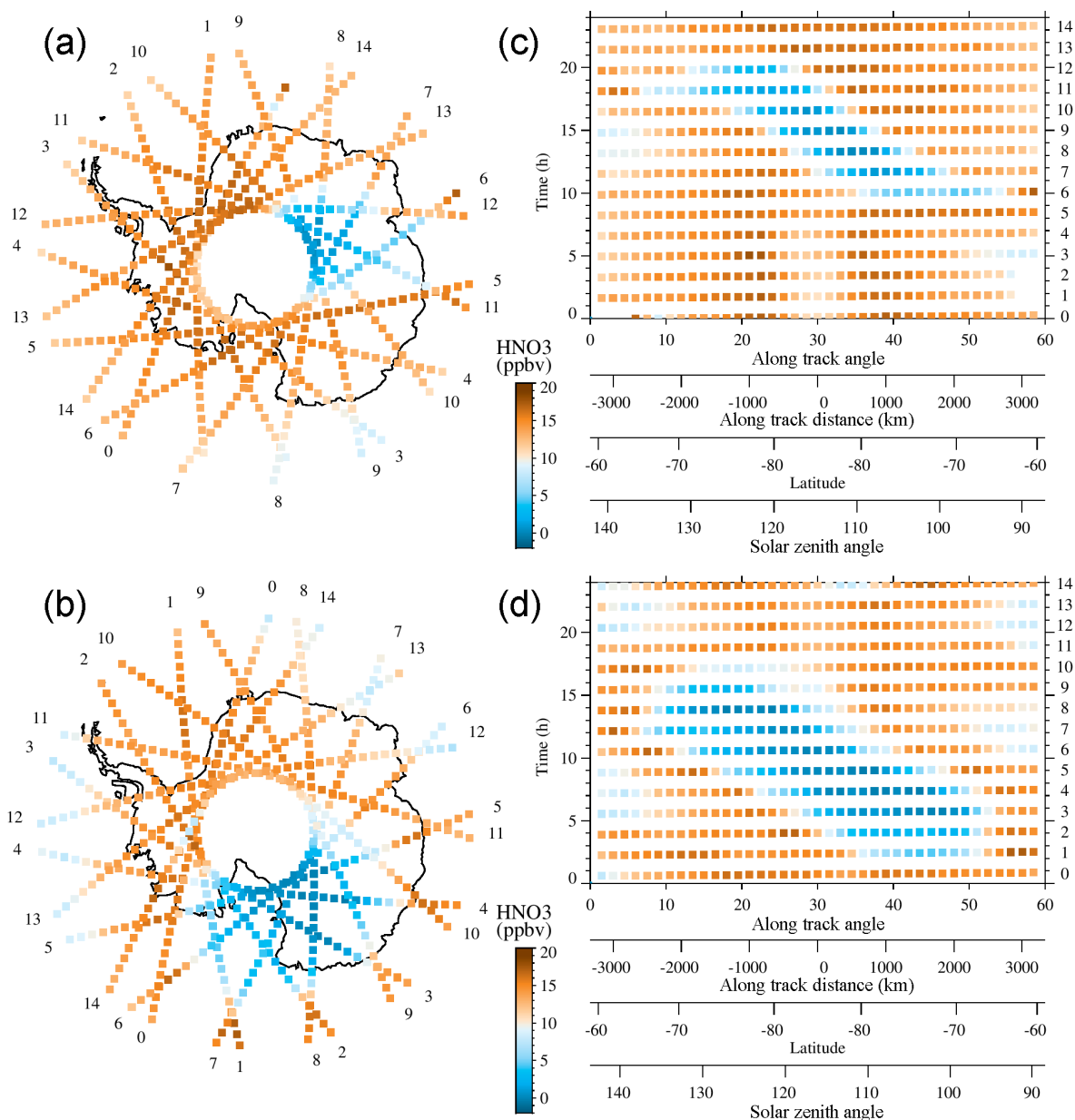

Figure 2. (a) Polar stereographic projection of the MLS measurement locations for all orbits over the Antarctic on $2009 \mathrm{~d} 146$ (26 May 2009 ). Each orbit is numbered sequentially. The square symbols denote the latitude and longitude locations of the MLS vertical profiles. The size of the squares is not representative of the along-track or across-track resolutions. The $\mathrm{HNO}_{3}$ volume mixing ratio at $32 \mathrm{hPa}$ is given by the color bar. (c) The same data points are shown as a temporal raster plot. The ordinate is the time of day in hours (UT) and the abscissa is the geodetic along-track angle. The squares denote the time of day of the MLS measurement and the measurement location with respect to the closest approach of the orbit track to the South Pole. Each orbit is numbered along the right ordinate. Also shown are the along-track distance, the latitude, and the solar zenith angle. The size of the squares is not representative of the MLS integration time or the along-track resolution. (b, d) As for (a, c), except for 2009d156 (5 June 2009).

of the MLS $\mathrm{HNO}_{3}, \mathrm{CALIOP}$ L2PSCMask, and the smoothed total and perpendicular backscatter ratios. Additionally, inspection of the MLS gas-phase $\mathrm{HNO}_{3}$ identifies a coincident decrease also consistent with the location of the CALIOP PSCs (note that the ZOMM model $\mathrm{HNO}_{3}$ is not shown by Hoyle et al., 2013).

\subsection{Modeled uptake of $\mathrm{HNO}_{3}$, lidar backscatter, and infrared extinction in PSCs}

We model the microphysics of representative STS and NAT particle distributions according to the methodology given in Pitts et al. (2009) and Lambert et al. (2012). For the lidar scattering calculations, Mie theory is used for liquid spherical particles and the T-matrix (Mishchenko and Travis, 1998) for solid NAT particles. As we noted in Lambert et al. (2012), the NAT particle shape is an open issue, and we continue here to use a range of spheroidal shapes to illustrate the lidar sensitivity to NAT. Real refractive indices at $532 \mathrm{~nm}$ were assumed to be 1.43 for STS and 1.50 for NAT, with zero imaginary refractive indices for both particle types. For the mid-infrared region, complex refractive indices were obtained from the tabulations given by Myhre et al. (2005) for STS and Toon et al. (1994) for NAT.

Existence temperatures of the PSC types are calculated using equilibrium thermodynamics and are dependent on the ambient partial pressures of $\mathrm{H}_{2} \mathrm{O}$ in the case of the ice frost point, $T_{\text {ICE }}$ (Murphy and Koop, 2005), and also $\mathrm{HNO}_{3}$ for $T_{\mathrm{NAT}}$ (Hanson and Mauersberger, 1988) and STS 

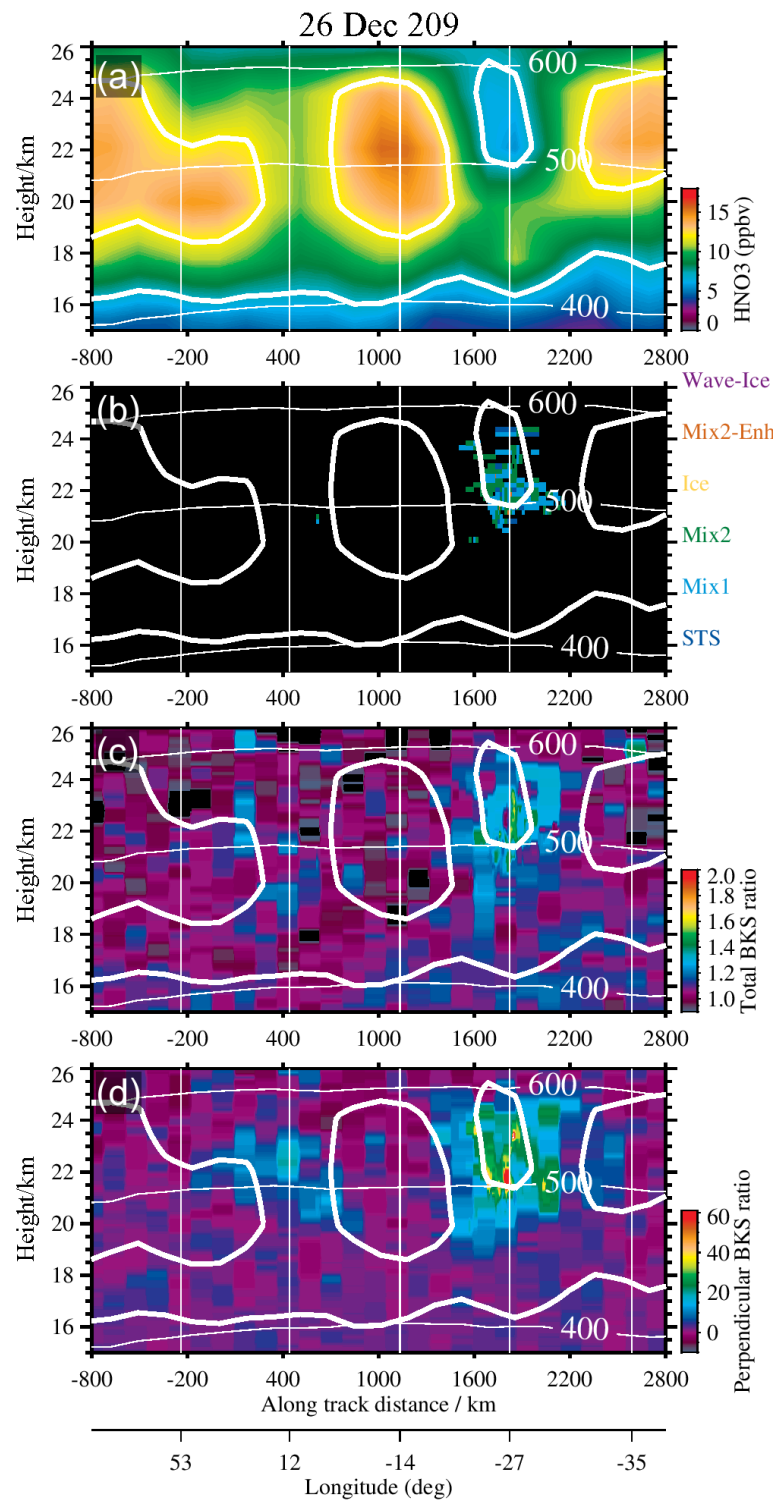

Figure 3. Comparison of along-track data for the partial orbit shown by Hoyle et al. (2013) (note that the $x$ axis is reversed here from their figure). (a) $\mathrm{MLS} \mathrm{HNO}_{3}$ showing sequestration at 12 and $-27^{\circ}$ longitude. (b) CALIOP PSC Mask does not show detection of PSCs at $12^{\circ}$ longitude. (c) Smoothed CALIOP total backscatter ratio. (d) Smoothed CALIOP perpendicular backscatter ratio. Solid

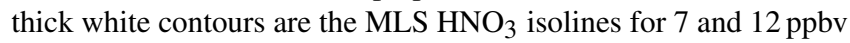
$\mathrm{HNO}_{3}$. Solid thin white vertical lines are the longitude markers shown by Hoyle et al. (2013). The detection of PSCs near $12^{\circ}$ longitude is evident in the smoothed CALIOP perpendicular backscatter ratio along with the corresponding $\mathrm{HNO}_{3}$ sequestration measured by MLS in (a).

(Carlsaw et al., 1995). Errors in the calculations of these reference temperatures arising from uncertainties in the MLS $\mathrm{H}_{2} \mathrm{O}$ and $\mathrm{HNO}_{3}$ data are estimated to be $\leq 0.5 \mathrm{~K}$ for $T_{\mathrm{ICE}}$ and $\leq 0.7 \mathrm{~K}$ for $T_{\mathrm{NAT}}$ in the pressure range $70-20 \mathrm{hPa}$.
SBS particles grow by condensation on cooling, first by uptake of $\mathrm{H}_{2} \mathrm{O}$ from the gas phase and, then, at sufficiently low temperatures, uptake of $\mathrm{HNO}_{3}$ occurs, forming STS at a few kelvin below the NAT point close to $T_{\mathrm{STS}} \sim T_{\mathrm{NAT}}-$ 3.5 K (Carslaw et al., 1997; Drdla et al., 2003). In the polar stratosphere, NAT is thermodynamically stable at temperatures below $T_{\mathrm{NAT}} \sim T_{\mathrm{ICE}}+7 \mathrm{~K}$, although the NAT nucleation process is still not understood in detail.

The PSC detection limits for lidar backscatter and infrared extinction are dependent on the background aerosol loading in addition to measurement noise. Under conditions of high quiescent background aerosol loadings (e.g., at times perturbed by volcanic aerosol), a higher threshold is required to discriminate PSCs from the background. The thresholds also depend on vertical and horizontal averaging both along-track and across-track. In the case of MLS, the across-track averaging (i.e., the $240 \mathrm{GHz}$ antenna beam width at the tangent point) is around $8 \mathrm{~km}$, the vertical field of view for $\mathrm{HNO}_{3}$ is a few kilometers and the along-track sampling is over several hundred kilometers. We have investigated coarser averaging of the lidar data in the along-track and vertical directions to achieve better signal-to-noise. However, the across-track averaging of CALIOP is only about $0.09 \mathrm{~km}$, and as a result the sampling volumes of limb sounding instruments are 3 or more orders of magnitude larger. The nominal sampling volumes that demonstrate the much larger limb sounder sampling are shown in Table 1.

For illustration, here we use typical threshold levels appropriate for the CALIOP Level-1b v3 lidar data gridded with a $50 \mathrm{~km}$ by $0.5 \mathrm{~km}$ resolution (Lambert et al., 2012): total backscatter ratio, $R_{\mathrm{T}}=1.25$ (STS type threshold), and perpendicular backscatter, $\beta_{\perp}=2.5 \times 10^{-6} \mathrm{~km}^{-1} \mathrm{sr}^{-1}$ (MIX type threshold). For a mid-infrared limb sounder operating in the window region near $12 \mu \mathrm{m}$ at $46 \mathrm{hPa}$, we use an extinction threshold, $k_{\mathrm{ext}}=5 \times 10^{-5} \mathrm{~km}^{-1}$. This value is based on measurements by the Improved Stratosphere and Mesosphere Sounder (Lambert et al., 1996) poleward of $70^{\circ} \mathrm{N}$ taken during late 1991, before the meridional transport of volcanic aerosol impacted polar background levels. We also found a similar extinction threshold by examining the characteristics of the polar aerosol extinction measured by the High Resolution Dynamics Limb Sounder (HIRDLS) (Massie et al., 2007) during 2005-2007. For both ISAMS and HIRDLS the distribution of the background aerosol extinction is modeled adequately by a log-normal distribution, with the stated threshold being at least 3 standard deviations higher than the mean of the extinction distribution. For the Michelson Interferometer for Passive Atmospheric Sounding (MIPAS) instrument, Spang and Remedios (2003) determined a cloud-index (CI) parameter with a threshold for PSC detection of $\mathrm{CI}<4.5$, and Höpfner et al. (2006b) indicated that this corresponds to a volume density detection limit of 0.2 $0.4 \mu^{3} \mathrm{~cm}^{-3}$. Finally, the threshold level for detection of the gas-phase removal resulting from the ambient $\mathrm{HNO}_{3}$ uptake 
Table 1. Comparison of limb sounder and lidar sampling volumes.

\begin{tabular}{lrr}
\hline Dataset & $\begin{array}{r}\text { Sampling dimensions (km) } \\
\text { along-track vs. vertical vs. across-track }\end{array}$ & $\begin{array}{r}\text { Sample volume } \\
\left(\mathrm{km}^{3}\right)\end{array}$ \\
\hline CALIOP gridded L1B data & $50 \times 0.5 \times 0.09$ & 2.25 \\
CALIOP lowest-resolution L2PSCMask & $135 \times 0.18 \times 0.09$ & 2.19 \\
CALIOP highest-resolution L2PSCMask & $5 \times 0.18 \times 0.09$ & 0.081 \\
CALIOP regridded L2PSCMask & $167 \times 2.1 \times 0.09$ & 31.2 \\
MLS HNO3 Level-2 & $400 \times 4 \times 8$ & 12800 \\
\hline
\end{tabular}

in STS is taken to be $1 \mathrm{ppbv}$, slightly higher than the $0.6 \mathrm{ppbv}$ MLS measurement uncertainty.

\subsubsection{Equilibrium STS}

Figure $4 \mathrm{a}$ shows the temperature variation of the modeled equilibrium thermodynamic properties of STS based on the Carlsaw et al. (1995) parameterization and the calculated lidar backscatter and $12 \mu \mathrm{m}$ infrared extinction assuming Mie theory. The calculations assume a pressure of $46 \mathrm{hPa}$, 5 ppmv $\mathrm{H}_{2} \mathrm{O}$, and 12 ppbv total $\mathrm{HNO}_{3}$. In situ observations of the STS aerosol volume are well modeled (Carlsaw et al., 1994), as is the uptake of $\mathrm{HNO}_{3}$ from the gas phase (Lambert et al., 2012). Uptake of $\mathrm{HNO}_{3}$ from the gas phase varies rapidly $(50,10,1 \%)$ over a narrow temperature range $\left(T-T_{\mathrm{ICE}}=2.3,3.1,3.9 \mathrm{~K}\right)$, and the maximum temperature derivative $\left(-6.7 \mathrm{ppbv} \mathrm{K}^{-1}\right)$ is at $T-T_{\mathrm{ICE}}=2.9 \mathrm{~K}$. The theoretical residual gas-phase $\mathrm{HNO}_{3}$, accounting for the uptake in STS, is shown in Fig. $4 \mathrm{~b}$ as a function of the total backscatter and $12 \mu \mathrm{m}$ infrared extinction. In a scatter plot, the observations of $\mathrm{HNO}_{3}$ and backscatter (or extinction) in the presence of STS are expected to lie beneath the theoretical curve, and this is investigated in Sect. 3.2. Figure 4c shows that the infrared extinction is marginally more sensitive to STS than the lidar backscatter, since the corresponding threshold equivalent condensed $\mathrm{HNO}_{3}$ contents of STS are 0.65 and $0.84 \mathrm{ppbv}$ for the two measurement approaches, respectively.

\subsubsection{STS/NAT mixtures}

The morphology of NAT particles is still an open question, as is the compactness of the particles (Molleker et al., 2014; Woiwode et al., 2014, 2016). Light scattering studies have repeatedly shown that detailed particle morphology cannot be deduced from the depolarization; e.g., Nousiainen et al. (2012) investigated simple and complex shapes with size parameters (ratio of the particle circumference to the wavelength) in the range 2-12 and real refractive indices in the range $1.55-1.603$ representative of silicate particles, and noted similar depolarization ranges for the fifteen different shapes (regular and irregular) that were analyzed. Therefore, by analogy, the selection of a few aspect ratios for a simple spheroidal shape is sufficient to demonstrate the variations in lidar backscatter properties and to highlight
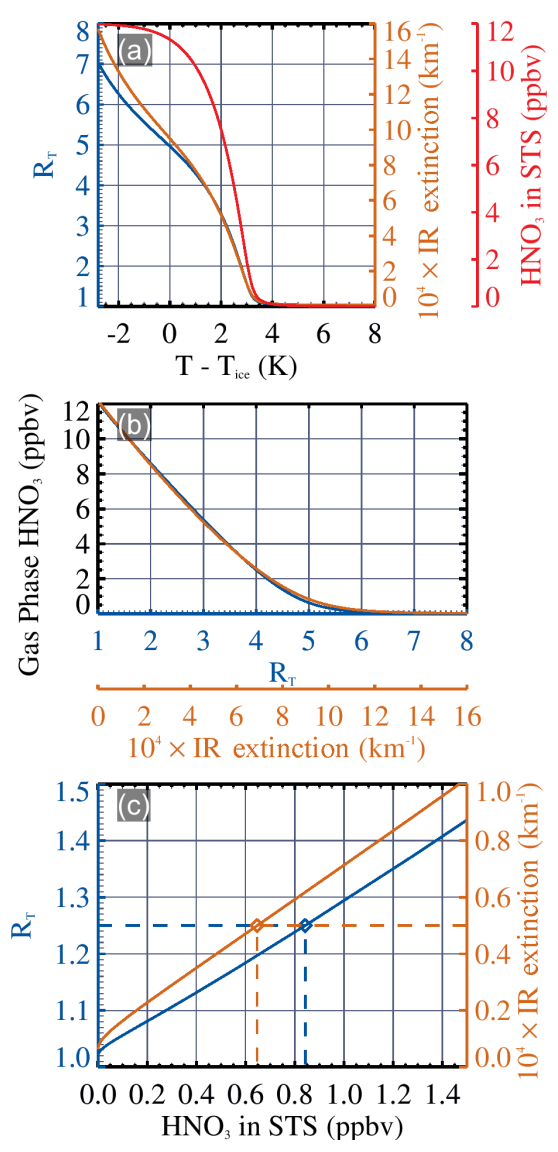

Figure 4. (a) Temperature variation relative to the frost point of the uptake of $\mathrm{HNO}_{3}$ in STS (red), the calculated $532 \mathrm{~nm}$ lidar total backscatter ratio, $R_{\mathrm{T}}$, (blue), and the $12 \mu \mathrm{m}$ infrared extinction (orange). (b) Gas-phase $\mathrm{HNO}_{3}$ vs. $532 \mathrm{~nm}$ total backscatter ratio (blue) and $12 \mu \mathrm{m}$ infrared extinction (orange). (c) STS detection limits for lidar backscatter ratio (blue diamond) and infrared limb extinction (orange diamond) and correspondence to the uptake of $\mathrm{HNO}_{3}$ in STS.

the challenges for lidar measurements to detect low number density large particle radii NAT. The NAT particles are modeled as spheroids with diameter-to-lengths (aspect ratios), $\epsilon$, both oblate $(1.2)$ and prolate $(0.8,0.9,0.95)$ using T-matrix calculations assuming a power-law NAT size distribution; otherwise, the STS/NAT mixtures are modeled as 
in Pitts et al. (2009) and Lambert et al. (2012). Previous investigations (Liu and Mishchenko, 2001; Flentje et al., 2002; Lambert et al., 2012) have noted that larger depolarizations (over $60 \%$ ) result from the more nearly spherical particles in the aspect ratio range $\epsilon=0.90-1.10$. Recent analyses of the CALIOP data (Engel et al., 2013; Hoyle et al., 2013) have used $\epsilon=0.90$ for NAT to improve modeling of the observed CALIOP depolarization range. Note that although the total backscatter is often dominated by STS, the use of a perpendicular backscatter threshold (Lambert et al., 2012; Pitts et al., 2013), rather than an aerosol depolarization threshold, reduces the possibility of the STS signal to mask the NAT signal in STS/NAT mixtures (Biele et al., 2001).

The large particle example shown in Fig. 5a is for a NAT particle distribution with a number density $N_{\mathrm{NAT}}=$ $0.001 \mathrm{~cm}^{-3}$ and effective radius $R_{\text {eff }}=6.5 \mu \mathrm{m}$. Note that in this example the uptake of $\mathrm{HNO}_{3}$ follows the NAT equilibrium curve until the saturation point is reached and the condensed $\mathrm{HNO}_{3}$ equals the volume in the assumed NAT particle distribution (plateau region with $4 \mathrm{ppbv}$ condensed $\mathrm{HNO}_{3}$ ). No further uptake of $\mathrm{HNO}_{3}$ occurs until the temperature decreases sufficiently to allow growth of STS. This example is a crude, but not unrealistic, snapshot of a possible STS/NAT mixture at a particular time because the growth of NAT is kinetically limited (Voigt et al., 2005). A high number density of NAT nuclei would ultimately lead to a NAT distribution with smaller particle sizes than a low number density since the available $\mathrm{HNO}_{3}$ is spread over a large number of particles (Jensen et al., 2002). Once nucleated, a NAT particle will continue to grow, provided there is sufficient $\mathrm{HNO}_{3}$ and $\mathrm{H}_{2} \mathrm{O}$ available and $T<T_{\mathrm{NAT}}$ such that the $\mathrm{HNO}_{3}$ vapor pressure over NAT is supersaturated, until it attains its equilibrium size (reaching a radius of tens of microns). Gravitational sedimentation may cause the NAT particles to descend into a region of lower $\mathrm{HNO}_{3}$ and/or rising temperature, causing evaporation rather than growth. The Wegener-BergeronFindeisen process will cause sequestration of $\mathrm{HNO}_{3}$ by NAT in a mixed-phase STS/NAT cloud at the expense of the $\mathrm{HNO}_{3}$ in the liquid STS (Voigt et al., 2005). However, if the STS forms quickly by rapid cooling, then the uptake of ambient $\mathrm{HNO}_{3}$ can be predominantly into STS rather than into NAT. Growth of NAT is therefore retarded at these low temperatures of a few K above the frost point (Voigt et al., 2005).

The lidar detection of NAT (backscattering in the visible spectrum) is sensitive to the asphericity parameter, whereas the infrared extinction (dependent mainly on emission and therefore particle volume) is not. Hence, there are four lidar total backscatter curves corresponding to each aspect ratio in Fig. 5a, but only a single infrared extinction curve is plotted that is representative of all four. Likewise, the inferred uptake of $\mathrm{HNO}_{3}$ by STS/NAT as measured by microwave observations is also insensitive to particle shape (in addition, the aerosol emission is negligible in the microwave region and has no effect on the gas measurements). In the absence of an actual PSC detection, the observed reduction in gas-
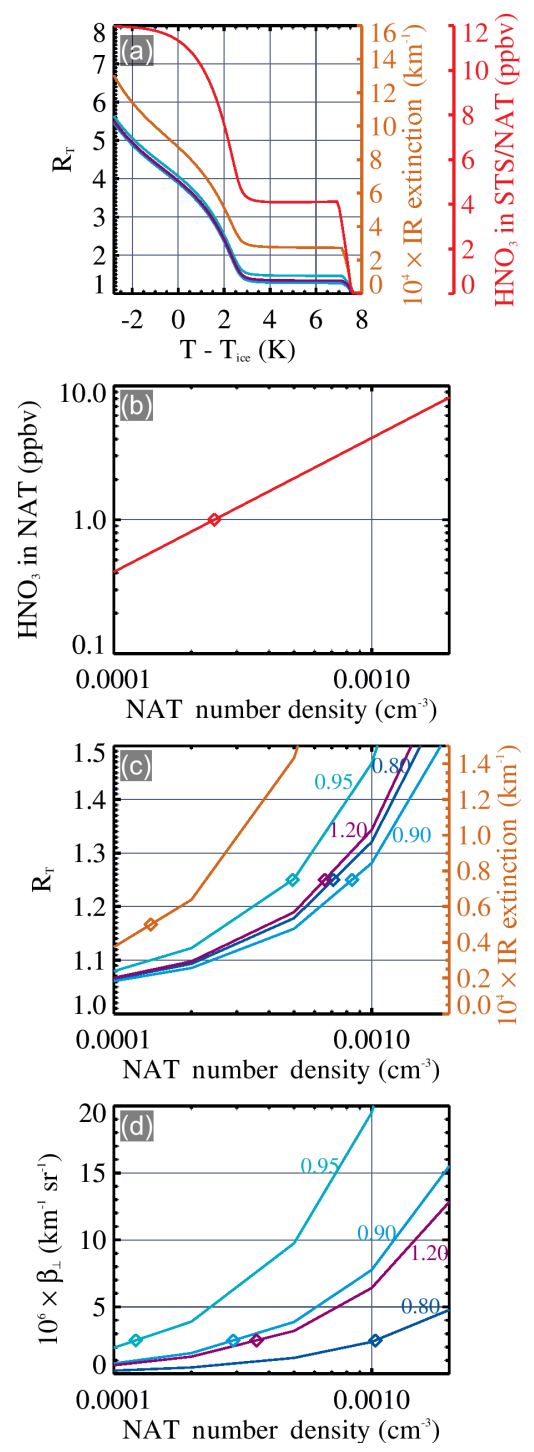

Figure 5. (a) Temperature variation relative to the frost point of the uptake of $\mathrm{HNO}_{3}$ in an STS/NAT mixture (red) for a NAT number density of $0.001 \mathrm{~cm}^{-3}$ and an effective radius of $6.5 \mu \mathrm{m}$, the calculated lidar backscatter ratio for four different particle shape aspect ratios (purple-blue), and the $12 \mu \mathrm{m}$ infrared extinction (orange). (b) Condensed $\mathrm{HNO}_{3}$ in NAT (red line) at $T-T_{\mathrm{ICE}}=5 \mathrm{~K}$ as a function of NAT number density. (c) NAT detection limits for lidar total backscatter ratio (blue-purple diamonds) and infrared limb extinction (orange diamond) and correspondence to the NAT number density. (d) As (c), except for the lidar perpendicular backscatter coefficient only.

phase $\mathrm{HNO}_{3}$ is an indirect detection method of PSC activity (Lambert et al., 2012), since either the $\mathrm{HNO}_{3}$ is condensed into NAT PSCs below the detection threshold of the lidar or the NAT particles were so large that they sedimented and the missing gas-phase $\mathrm{HNO}_{3}$ is the result of permanent denitrification. 
The theoretical condensed $\mathrm{HNO}_{3}$ in NAT at a fixed temperature of $T-T_{\mathrm{ICE}}=5 \mathrm{~K}$ is shown in Fig. $5 \mathrm{~b}$ as a function of the NAT number density. At this temperature, the STS contribution to the backscatter, infrared extinction, and $\mathrm{HNO}_{3}$ uptake is negligible (see Fig. 4) and we may safely concentrate on the properties of the NAT particles alone. The temperature is about $2.5 \mathrm{~K}$ below the NAT existence temperature and about $2 \mathrm{~K}$ above the temperature at which substantial uptake of $\mathrm{HNO}_{3}$ into STS occurs. The red diamond symbol marks the detection threshold for $1 \mathrm{ppbv}$ of $\mathrm{HNO}_{3}$ condensed in NAT (or equivalently a 1 ppbv uptake of $\mathrm{HNO}_{3}$ from the gas phase) and corresponds to a NAT number density of $2.45 \times 10^{-4} \mathrm{~cm}^{-3}$. In Fig. $5 \mathrm{c}$ we show the total lidar backscatter and infrared extinction as a function of the NAT number density. The total backscatter detection threshold (1.25) for the four particle aspect ratios corresponds to NAT number densities ranging from 4.9 to $8.4 \times 10^{-4} \mathrm{~cm}^{-3}$ compared to $1.4 \times 10^{-4} \mathrm{~cm}^{-3}$ for the infrared extinction threshold $\left(k_{\mathrm{ext}}=5 \times 10^{-5} \mathrm{~km}^{-1}\right)$. For this large particle radius example, uptake of $\mathrm{HNO}_{3}$ and infrared detections are more sensitive than the lidar total backscatter. The perpendicular backscatter coefficient (Fig. 5d) is more sensitive than the total backscatter to the presence of non-spherical NAT, resulting in the detection of lower number densities ranging from 1.2 to $3.6 \times 10^{-4} \mathrm{~cm}^{-3}$, except for the 0.8 aspect ratio, which shows less sensitivity. Hence, operation of a lidar with an orthogonal polarization channel can substantially improve the detection threshold for non-spherical NAT for some aspect ratios. The infrared detection is shown to be more sensitive for the large particle range, except for an aspect ratio of 0.95 for which the sensitivities are comparable.

\subsubsection{Intercomparisons of PSC detection techniques}

The sensitivity of different techniques employed to detect PSCs over a wide range of number densities and effective radii is illustrated with Fig. 6. Selected detection limits for CALIOP lidar, infrared limb emission, and inferred detection by the measured uptake of $\mathrm{HNO}_{3}$ from the gas phase or the in situ aerosol detection of condensed $\mathrm{HNO}_{3}$ in NAT are shown. Again, the calculations are for a temperature $T-T_{\mathrm{ICE}}=5 \mathrm{~K}$, since the purpose of the comparison is to show the potentially large variation in lidar backscatter response to NAT PSCs of differing asphericities. The lines in Fig. 6 mark the detection limits for the various techniques and indicate the lowest NAT number density $\left(N_{\mathrm{NAT}}\right)$ that can be detected for a given NAT effective radius $\left(R_{\text {eff }}\right)$; i.e., any combination of $\left(N_{\mathrm{NAT}}, R_{\mathrm{eff}}\right)$ lying below a given line is below the detection limit for that particular technique. The gray shading indicates the region that is below the detection threshold for any of the techniques assuming the given variation in NAT aspect ratio. The solid (dashed) purple-blue lines correspond to the detection thresholds for total backscatter (perpendicular backscatter) for different aspect ratios. As expected from the previous section, the perpendicular backscatter is in general more

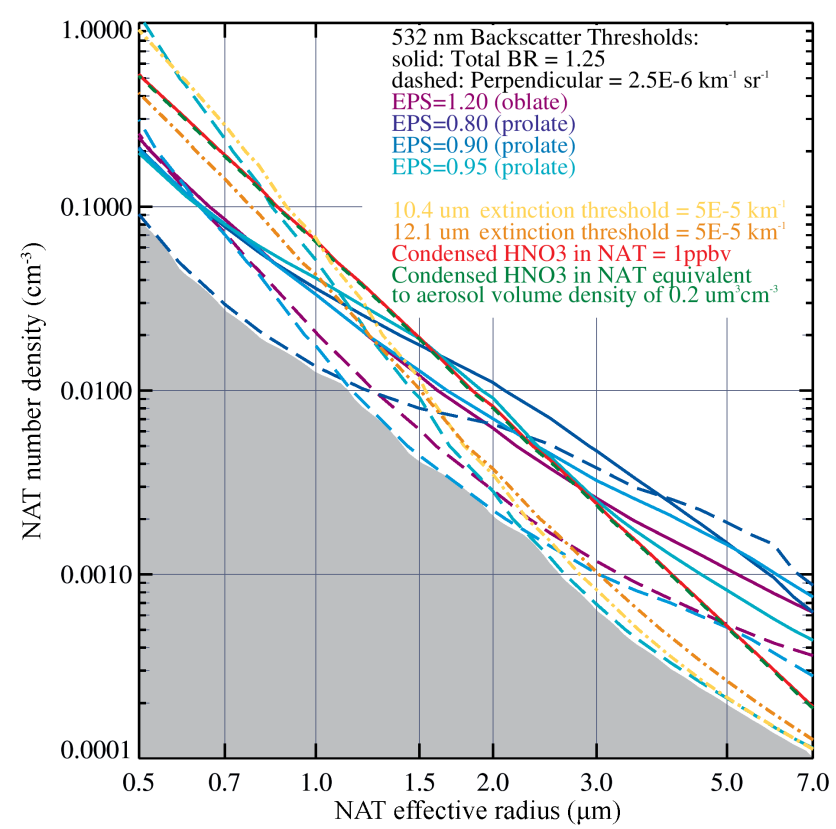

Figure 6. Intercomparison of the sensitivity of various PSC detection techniques to a range of NAT number densities and effective radii at a temperature of $T-T_{\mathrm{ICE}}=5 \mathrm{~K}$, for an ambient pressure of $46 \mathrm{hPa}, 5 \mathrm{ppmv} \mathrm{H}_{2} \mathrm{O}$, and $12 \mathrm{ppbv}$ total $\mathrm{HNO}_{3}$. The purple-blue lines indicate the limits of detection for lidar with total backscatter (solid) and orthogonal channel (dashed) for a range of aspect ratios (EPS). The red (green) line indicates the sensitivity of an in situ sampling instrument to $1 \mathrm{ppb}$ of condensed $\mathrm{HNO}_{3}$ in NAT (aerosol density, $0.2 \mu \mathrm{m}^{3} \mathrm{~cm}^{-3}$ ) or the equivalent uptake from the gas phase (e.g., by a microwave limb sounder). Yellow/orange lines indicate the sensitivities in the mid-infrared for a limb sounder at two wavelengths. NAT PSCs with particle distribution characteristics lying within the gray shaded region are undetectable by any of the above techniques, with the assumed horizontal and vertical averaging scales given in the text.

sensitive to the presence of low number density NAT than is the total backscatter ratio. However, there is substantial variation in the sensitivity to the particle aspect ratio, $\epsilon$. The sensitivity curves for infrared extinction (yellow) and lidar perpendicular backscatter are similar for the $\epsilon=0.95$ case for $R_{\text {eff }} \geq 4$, whereas for $\epsilon=0.8$ the lidar is much less sensitive. For low number density/large particle NAT (bottom right of Fig. 6), the uptake of $\mathrm{HNO}_{3}$ from the gas phase (red) can still be quite substantial (1 ppbv) and is independent of the particle asphericity. The extremes of the lidar sensitivity to small NAT $\left(R_{\text {eff }}<1 \mu \mathrm{m}\right)$ (ranging over an order of magnitude in particle number density) are seen to be reversed for large NAT $\left(R_{\text {eff }}>5 \mu \mathrm{m}\right)$; i.e., the lidar technique is more sensitive to small NAT with an aspect ratio of 0.80 than 0.95 and vice versa for large NAT. Except for the aspect ratio of 0.95 , the detection limits for infrared emission and the uptake of 1 ppbv $\mathrm{HNO}_{3}$ from the gas phase become more sensitive than the lidar limit for large NAT with $R_{\text {eff }}>2.5$ and 
$R_{\text {eff }}>5 \mu \mathrm{m}$, respectively. The near coincidence of the green dashed line and red solid line shows that a NAT volume density of $0.2 \mu \mathrm{m}^{3} \mathrm{~cm}^{-3}$ (limit of detection for MIPAS given by Höpfner et al., 2006b) is approximately equivalent to $1 \mathrm{ppbv}$ of condensed $\mathrm{HNO}_{3}$. This threshold is consistent with a previous study by Höpfner (2004) that showed that large particle, small number density NAT can be detected by MIPAS. The specific case studied was for a particle distribution with a $7 \mu \mathrm{m}$ mode radius and number density $2.9 \times 10^{-4} \mathrm{~cm}^{-3}$, which we estimate to be equivalent to a $12 \mu \mathrm{m}$ extinction of $1.9 \times 10^{-4} \mathrm{~km}^{-1}$ (practically independent of the particle aspect ratio in the infrared region).

\subsection{Separation of PSC types using backscatter, $\mathrm{HNO}_{3}$, and temperature}

In this section we investigate the lidar PSC classification by exploring the 2-D cross sections resulting from projections of the 3-D coordinate space of temperature, $\mathrm{HNO}_{3}$, and total backscatter. Here we use the CALIOP Level-1b v3 data with coincident MLS data processed as detailed in Lambert et al. (2012) for the period 10 May to 25 October 2009 in the Antarctic at 46-21 hPa. In Fig. 7 we show probability density functions (PDFs) classified according to the CALIOP PSC scheme (Pitts et al., 2009), with modifications discussed in Lambert et al. (2012) and Pitts et al. (2013), in six columns (ALL, LIQ, MIX1, MIX2, ICE, and None) and four rows (described below). The ALL class is the sum of the individually classified PSC components LIQ, MIX1, MIX2, and ICE. The None class represents all cases below the CALIOP detection threshold. The four rows for each column show the depolarization vs. normalized backscatter and the corresponding PDFs for the three possible combinations of pairings from the temperature, $\mathrm{HNO}_{3}$, and total backscatter coordinates in the PSC classification. Nitric acid vs. temperature has been shown previously by Lambert et al. (2012) and Pitts et al. (2013) (and is included for completeness), but backscatter vs. temperature and $\mathrm{HNO}_{3}$ vs. backscatter are shown for the first time here.

\subsubsection{Row 1: depolarization $(\delta)$ vs. normalized backscatter $\left(1-1 / R_{\mathrm{T}}\right)$}

CALIOP data analysis, detection, and classification are discussed in Lambert et al. (2012) and Pitts et al. (2013). The PSC classes are shown in the CALIOP depolarization vs. backscatter classification diagram in the first row. Black solid lines indicate the main PSC types. Class boundaries for MIX2-enh and wave ice $\left(R_{\mathrm{T}}>50\right)$ are shown as black dashed lines, but are not differentiated here from the MIX2 and ICE main classes. The classification boundaries were originally chosen (Pitts et al., 2009) to distinguish STS (depolarization less than 3\%), STS/NAT mixtures (significant depolarization indicating a solid component), and ice. Note that the LIQ/MIXI class boundary is fuzzy, and depolarization values (the ratio of the perpendicular to parallel backscatter) can exceed $3 \%$ for LIQ because of measurement noise even though the perpendicular backscatter component indicates below-threshold response. Similarly, the None class boundary is fuzzy because of measurement noise. The black-white dashed line shows the theoretical lower limit of detection as a locus of points $(\delta$, $1-1 / R_{\mathrm{T}}$ ) for the chosen perpendicular backscatter threshold, $\beta_{\perp}=2.5 \times 10^{-6} \mathrm{~km}^{-1} \mathrm{sr}^{-1}$, calculated for a typical polar atmosphere from the expression

$$
R_{\mathrm{T}}(\delta)=\left[\left(1+\frac{1}{\delta}\right)\left(\beta_{\perp}-\beta_{\mathrm{m}_{\perp}}\right)+\beta_{\mathrm{m}}^{\mathrm{T}}\right] \frac{1}{\beta_{\mathrm{m}}^{\mathrm{T}}},
$$

where the molecular depolarization $\delta_{\mathrm{m}}$ is 0.0036 , the molecular perpendicular backscatter component $\beta_{\mathrm{m}_{\perp}}$ is $\beta_{\mathrm{m}}^{\mathrm{T}} \frac{\delta_{\mathrm{m}}}{1+\delta_{\mathrm{m}}}$, the total Rayleigh scattering (both polarizations) is $\beta_{\mathrm{m}}^{\mathrm{T}}$, and the fractional depolarization range is $\delta=0 \ldots 1$. This low detection limit is not strictly attained in practice because of additional spatial coherence constraints that are imposed to reduce false positives to less than $0.1 \%$ (Lambert et al., 2012). The coherence constraint results in the distribution of points in the None class appearing to the right side of the black-white dashed line. All the other imposed class boundaries are sharp, although this does not imply that the distinction between these PSC types is definitive. For example, as noted in Pitts et al. (2013), the ICE "arm" close to the LIQ class (normalized backscatter 0.7-0.85) is intersected by the MIX2 / ICE boundary. Better separation between the MIX2 and ICE classes based on allowing for the seasonal variation in the location of the ice "arm" associated with denitrification was discussed in Pitts et al. (2013). Overall, the classification using the 2-D regions of the depolarization vs. normalized backscatter provides very good discrimination between STS and solid-particle PSCs.

\subsubsection{Row 2: Gas-phase $\mathrm{HNO}_{3}$ vs. $T-T_{\text {ICE }}$}

In Lambert et al. (2012) and Pitts et al. (2013), we demonstrated that the uptake of $\mathrm{HNO}_{3}$ vs. temperature, classified according to CALIOP PSC types, is in good agreement with expected temperature existence regimes for STS, NAT, and ice, except for an apparent $\sim 1 \mathrm{~K}$ bias with respect to the GEOS-5 temperatures. This is consistent with comparisons (not shown) of coincident independent temperatures obtained from the Constellation Observing System for Meteorology, Ionosphere, and Climate (COSMIC) GPS Radio Occultation data, which indicate a cold bias in GEOS- 5 of up to $0.7 \mathrm{~K}$ in the 2009 Antarctic lower stratosphere. The LIQ class follows the theoretical equilibrium uptake of $\mathrm{HNO}_{3}$ by STS (blue lines from 2 to $24 \mathrm{ppbv}$ in 2 ppbv steps), and the MIXI and MIX2 classes show significant non-equilibrium behavior, along with two discernible branches following the STS and the NAT (green line) equilibrium curves. The ICE class is a compact and roughly symmetric distribution, located at the lowest temperatures and lowest $\mathrm{HNO}_{3}$ gas-phase amounts. 

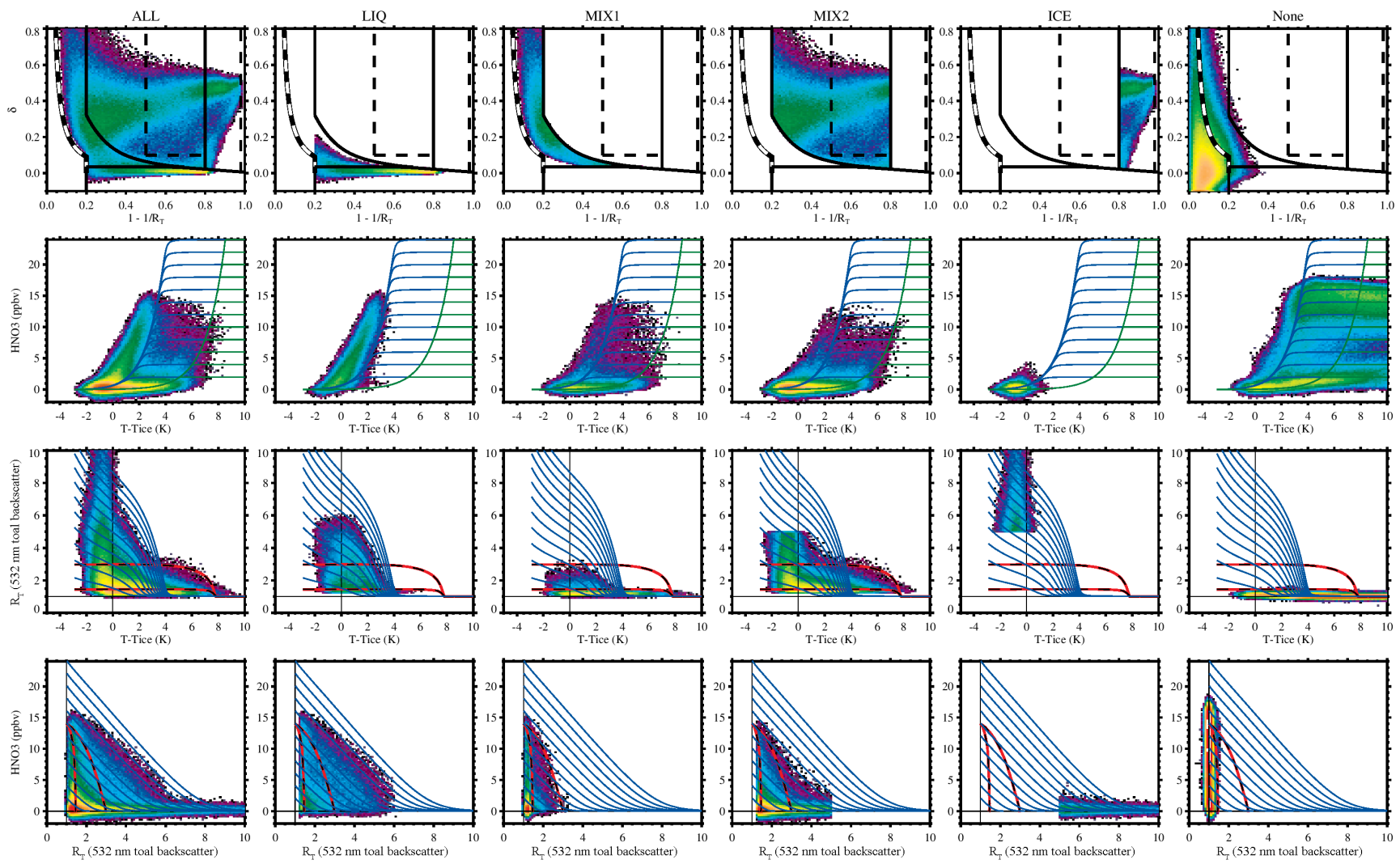

10

100

1000

Figure 7. CALIOP PSC type classifications and their corresponding 2-D cross-section pairs derived from the 3-D coordinate space of temperature $T-T_{\mathrm{ICE}}, \mathrm{HNO}_{3}$, and total backscatter, $R_{\mathrm{T}}$, for 10 May to 25 October 2009. The six columns are the CALIOP PSC types identified in the text. Blue (green) lines are theoretical calculations for total $\mathrm{HNO}_{3}$ from 2 to 24 ppbv in 2 ppbv steps for STS (NAT) equilibrium. Red-black dashed lines are theoretical calculations for equilibrium NAT with number densities $0.001 \mathrm{~cm}^{-3}$ (bottom curve) and $0.01 \mathrm{~cm}^{-3}$ (top curve) and $14 \mathrm{ppbv}$ total $\mathrm{HNO}_{3}$. The lower limit of detection, given by the black-white dashed line, is described in the text.

The leading edge of the $\mathrm{HNO}_{3}$ gas-phase distribution for the None class follows the STS uptake curve. Also visible is a separate highly denitrified branch $\left(\mathrm{HNO}_{3}<5 \mathrm{ppbv}\right)$ extending to beyond $10 \mathrm{~K}$ above the ice frost point.

\subsubsection{Row 3: total backscatter $\left(R_{\mathrm{T}}\right)$ vs. $T-T_{\mathrm{ICE}}$}

This row shows the temperature domains corresponding to the various CALIOP PSC classes (the region of highest backscatter, $R_{\mathrm{T}}>10$, is not shown). The blue lines indicate the theoretical STS backscatter vs. $T-T_{\mathrm{ICE}}$ for gasphase $\mathrm{HNO}_{3}$ increasing from 2 to $24 \mathrm{ppbv}$ in 2 ppbv steps. Note that the current depolarization/backscatter classification scheme does not use temperature as a discriminant. Backscatter vs. temperature is only used in the CALIOP classification scheme to determine daily detection thresholds (Pitts et al., 2009). The LIQ class shows a rapid increase in total backscatter near $T-T_{\mathrm{ICE}}=3.5 \mathrm{~K}$ (i.e., $T_{\mathrm{STS}}$ is located at the point the blue curves join the abscissa). There is a thin tail which does not reach out as far as $T_{\mathrm{NAT}}$ (located at the intersection of the two red-black dashed curves). The envelope of the theoretical STS curves (bounded by total $\mathrm{HNO}_{3}$ values of $16-18 \mathrm{pbbv}$ ) is a reasonable description of the distribution, but at the lowest temperatures and highest expected backscatter $(>6)$ the region is unpopulated. This may be because such conditions are favorable for freezing of STS to form ice (Koop et al., 1995). The MIXI class shows a tail with very low backscatter extending from $T_{\mathrm{STS}}$ out to beyond $T_{\mathrm{NAT}}$ and is consistent with $N_{\mathrm{NAT}} \leq 0.001 \mathrm{~cm}^{-3}$. At temperatures below $T_{\mathrm{STS}}$ the backscatter increases, but remains below values of about 3 , and the distribution is consistent with $N_{\text {NAT }} \leq 0.01 \mathrm{~cm}^{-3}$. The MIX2 class also shows a tail beyond $T_{\mathrm{NAT}}$ and is consistent with the largest $N_{\mathrm{NAT}}$ ranging up to slightly above $0.01 \mathrm{~cm}^{-3}$, except at the lowest temperatures below $T-T_{\mathrm{ICE}}=1 \mathrm{~K}$, where the backscatter increases rapidly. The demarcation between the MIX2 and ICE classes at $R_{\mathrm{T}}=5$ is somewhat arbitrary and leads to NAT/ice misclassification in this transition region. This effect is ameliorated by the use of a successive averaging scheme (Pitts et al., 2009), since ice can generally be detected at a higher spa- 
tial resolution than is shown here. At the highest backscatter values there is an apparent trend in the ICE class towards higher minimum temperatures. The None class shows a narrow distribution consistent with the chosen total backscatter threshold of 1.25 .

\subsubsection{Row 4: Gas-phase $\mathrm{HNO}_{3}$ vs. total backscatter $\left(\boldsymbol{R}_{\mathrm{T}}\right)$}

The blue lines indicate the theoretical STS $\mathrm{HNO}_{3}$ vs. backscatter curves. These show an almost linear decrease in $\mathrm{HNO}_{3}$ with increasing backscatter. For the LIQ class the regions $R_{\mathrm{T}}<2$ with high $\mathrm{HNO}_{3}$ and $R_{\mathrm{T}}>6$ with low $\mathrm{HNO}_{3}$ are not fully populated when compared to the theoretical curves. For low backscatter this is likely to indicate STS containing less than $0.8 \mathrm{ppbv}$ of $\mathrm{HNO}_{3}$, which cannot be detected. For high backscatter this may suggest the result of freezing of STS to ice (see Row 3 discussion). The LIQ class also shows a bulge in the PDF around $R_{\mathrm{T}}=4$ to 6 that reaches the theoretical curve for total $\mathrm{HNO}_{3}=22 \mathrm{ppbv}$. Since the None class indicates that the maximum $\mathrm{HNO}_{3}$ is 18.5 ppbv, the $\sim 3.5$ ppbv excess $\mathrm{HNO}_{3}$ in the LIQ PDF may have arisen from the formation of additional $\mathrm{HNO}_{3}$ produced from heterogeneous reactions occurring on the liquid particles and released into the gas phase or by renitrification from evaporation of sedimenting NAT clouds (see Sect. 4.2). The None class also indicates totally denitrified regions (consistent with the noise floor of the MLS measurements) with insufficient $\mathrm{HNO}_{3}$ to form any kind of non-ice PSCs. Note that the data in this row are independent of the suspected GEOS-5 temperature bias.

\section{Evaluation of CALIOP and MLS co-located measurements}

Although simultaneous co-located measurements of PSCs and gas constituents are obviously to be preferred over spatially and temporally decorrelated measurements, the availability of such measurements from MLS and CALIOP cannot be expected to provide full closure to the questions of PSC formation. Careful consideration of the details of PSC formation is required to reconcile the pieces of information garnered from the different measurement techniques. In this regard the ultimate aim would seem to be Lagrangian measurements following the full life-cycle of PSC evolution. However, further unresolved issues have emerged from the longduration stratospheric balloon flights by Ward et al. (2014), who describe measurements of the NAT nucleation rate that show much larger spatial inhomogeneities in NAT occurrence than anticipated.

\subsection{MLS and CALIOP orbit transects}

We have selected some views from the combined MLS and CALIOP data record to illustrate how the interpretation of the morphology of PSCs and gas-phase $\mathrm{HNO}_{3}$ in along-track transects is governed not only by the local ambient temperature, but also by the underlying temperature histories. Here we use the CALIOP Level-2 v1 PSC Mask dataset and also apply post-processing to generate coarser horizontal/vertical bins for a better comparison at the scale of the MLS alongtrack and vertical resolution. Each averaging bin is the size of the MLS along-track separation $(165 \mathrm{~km})$ and the height between the mid-points of the pressure levels $(2.1 \mathrm{~km})$ for the MLS $\mathrm{HNO}_{3}$ data product. Note that in this section we use the L2PSCMask data class name STS instead of LIQ.

In Fig. 8 we present along-track orbit transects in the 2009 Antarctic early winter. The four columns show a sample of orbit tracks over an 11-day period (day number/orbit number): 2009d136/11, 2009d145/6, 2009d145/13, 2009d147/9. The data in each row are (a) $\mathrm{MLS} \mathrm{HNO}_{3}$, (b) $T-T_{\mathrm{ICE}}$, (c) temperature history (TTE), (d) temperatures following the Lagrangian back-trajectories, (e) L2PSCMask CALIOP PSC classification, and (f) the post-processed Liquid/Solid index, LS_index $=(L-S) /(L+S)$, where $L$ is the number of observations in the STS classification and $S$ is any other PSC (solid) detection. The LS_index is the ratio of the number of CALIOP classifications occurring within the corresponding MLS along-track extent, with the extreme values of -1 indicating only solid class and +1 only liquid class detections. The LS_index represents the dominant PSC classification in a sample volume similar in size to the MLS gas species resolution. The pixel size is much larger than that in the L2PSCMask composition plot, and the composition speckle can be seen as "blocky" regions in the LS_index.

Several contour lines are superposed on the orbit transects: the black-white quasi-horizontal labeled contours show a sample of the MLS pressure levels $\left(\mathrm{HNO}_{3}\right.$ is retrieved at a six-level/decade change in pressure). The green and blue contours represent temperatures corresponding to the $T_{\mathrm{NAT}}$ threshold (using GEOS-5 and $\mathrm{MLS} \mathrm{HNO}_{3}, \mathrm{H}_{2} \mathrm{O}$ ) and $T_{\mathrm{ICE}}+$ $2 \mathrm{~K}$, respectively. The blue temperature contour encompasses an expected $\mathrm{HNO}_{3}$ uptake of about $50 \%$ from the gas phase into STS (see Fig. 4a). The yellow contour is the $\mathrm{HNO}_{3}$ 12 ppbv contour, and the red contour encloses the area with $\mathrm{TTE} \geq 3$ days.

Case 1: 16 May 2009/2009d136/11: $\mathrm{MLS} \mathrm{HNO}_{3}$ shows an extent of a few hundred kilometers with $\mathrm{HNO}_{3}$ uptake from the gas phase, which is offset from the region of the lowest local temperatures (Fig. 8b), but more closely located with peak values of TTE (Fig. 8c) (which are still below 3 days). CALIOP detects some pixels of MIX1 and fewer of MIX2 (Fig. 8e). Another orbit track (9, not shown) on the same day shows smaller $\mathrm{HNO}_{3}$ uptake with no coincident PSC detections. 

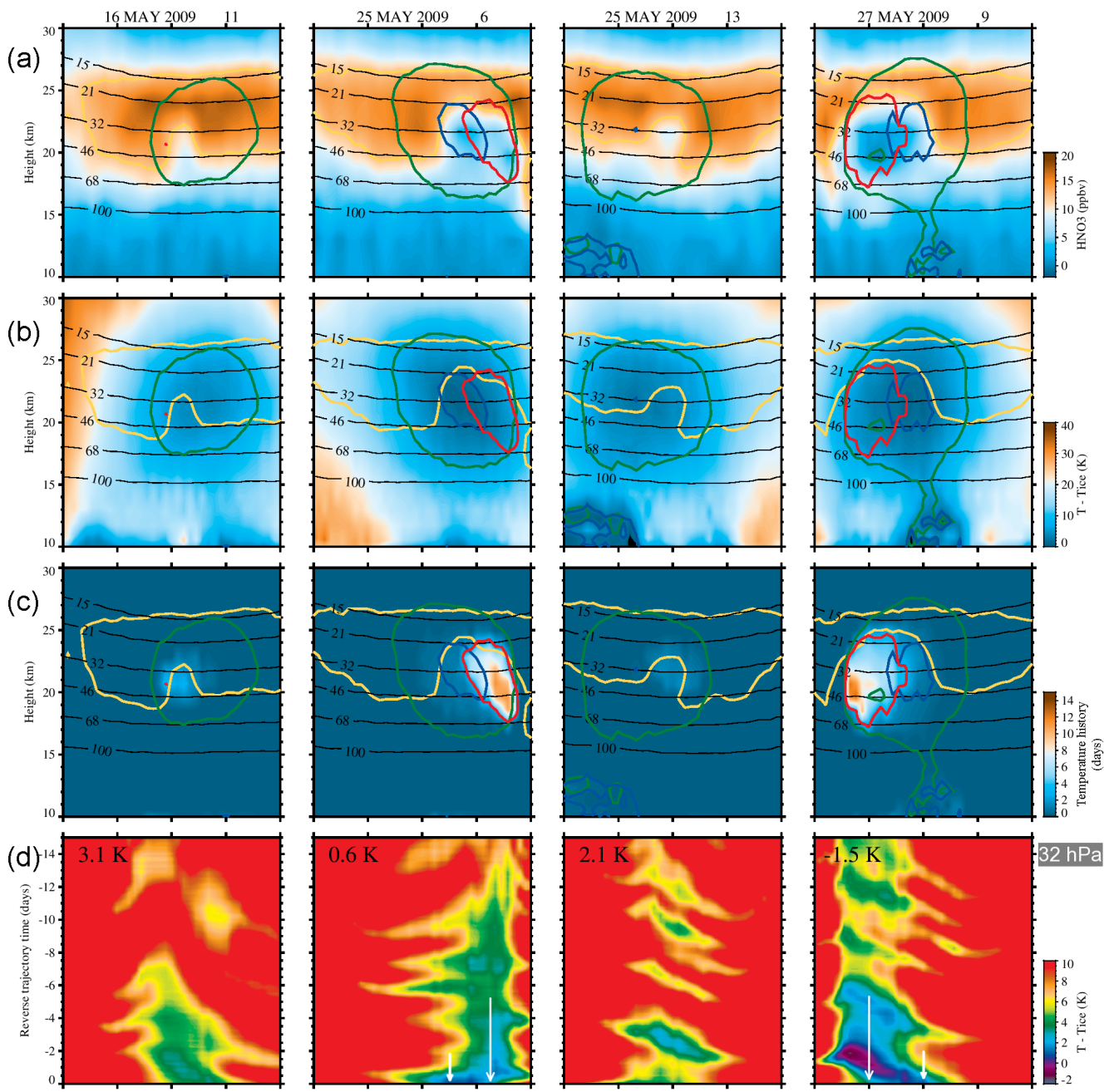

(e)
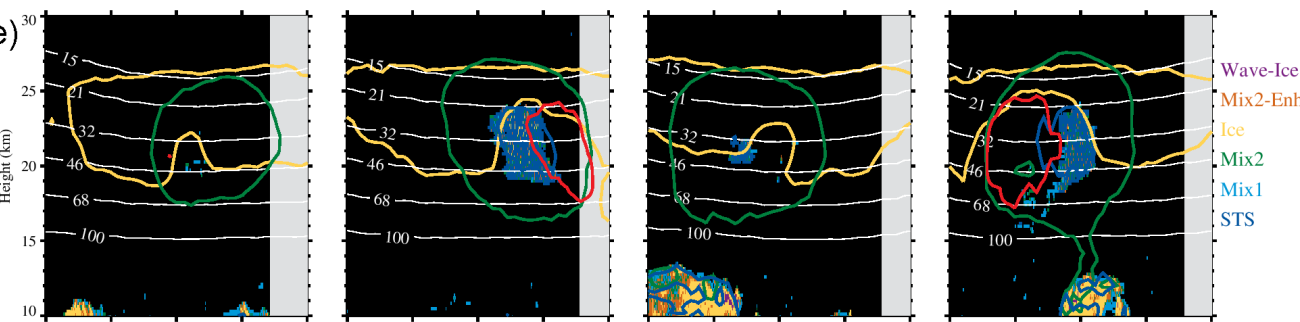

(f)
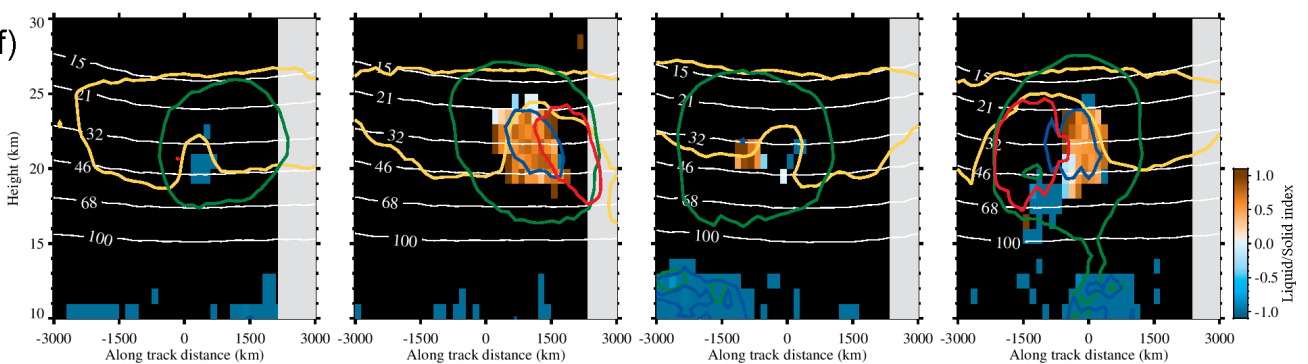

Figure 8. Co-located MLS and CALIOP orbit transects for selected orbits showing in six rows: (a) MLS HNO 3 . (b) Ambient temperature, $T-T_{\mathrm{ICE}}$. (c) Temperature threshold exposure, TTE (days). (d) Fifteen-day reverse trajectory temperature history, $T-T_{\mathrm{ICE}}$, ending at the $31 \mathrm{hPa}$ pressure level. Minimum temperature encountered along the trajectory is inset at the top left. (e) CALIOP L2PSCMask PSC classification. (f) LS_index. MLS pressure levels are shown as labeled black or white contours. Blue (green) contours indicate $T_{\text {ICE }}+2 \mathrm{~K}$ $\left(T_{\mathrm{NAT}}\right)$. Red contours indicate TTE values $\geq 3$ days. The MLS $12 \mathrm{ppbv} \mathrm{HNO}_{3}$ contours are indicated in yellow. Gray shading in (e, f) indicates that the CALIOP observations are in daylight. 
Case 2: 25 May 2009/2009d145/6: MLS $\mathrm{HNO}_{3}$ shows a substantial region of $\mathrm{HNO}_{3}$ uptake over $1500 \mathrm{~km}$ and suggests a combination of uptake in two separate regions, one located centrally within the local temperature minimum (blue contour, $T_{\mathrm{ICE}}+2 \mathrm{~K}$ ) and another offset extending to the right edge of the $T_{\mathrm{NAT}}$ contour (green). The temperature history (red contour) is the key to this rather apparent asymmetry of the $\mathrm{HNO}_{3}$ distribution with respect to the $T_{\mathrm{NAT}}$ contour, since the TTE clearly has larger values outside of the central local temperature minimum and is associated with the region of $\mathrm{HNO}_{3}$ uptake on the right. The L2PSCMask (Fig. 8e) shows a substantial STS cloud with some composition speckle, mainly coincident with the central local temperature minimum. The LS_index (Fig. 8f) shows predominantly liquid detections, with more solid detections at the top and lower-right edge of the cloud. The STS class (Fig. 8e) does not completely fill the local temperature contour on the right-hand side, which overlaps with the largest TTE values (red contour).

Case 3: 25 May 2009/2009d145/13: $\mathrm{MLS} \mathrm{HNO}_{3}$ shows significant uptake coincident with the peak temperature exposure history. The L2PSCMask (Fig. 8e) shows some MIX1/MIX2 class, but is not coincident with the largest $\mathrm{HNO}_{3}$ uptake. A very small area of local temperature minimum (blue contour), near the along-track distance coordinate at $-1000 \mathrm{~km}$ and on the $32 \mathrm{hPa}$ level, shows little $\mathrm{HNO}_{3}$ uptake and some STS class pixels.

Case 4: 27 May 2009/2009d147/9: MLS $\mathrm{HNO}_{3}$ indicates substantial $\mathrm{HNO}_{3}$ uptake (Fig. 8a) coincident with the local temperature minimum (blue contour), but also extending to the left edge of the $T_{\mathrm{NAT}}$ region (green contour). The greatest exposure to low temperatures (red contour) is associated with the left region of $\mathrm{HNO}_{3}$ uptake. The L2PSCMask (Fig. 8e) shows a substantial STS class (with multi-class speckle), but only in the right half of the minimum local temperature region. The left half is the area with the largest TTE values (red contour). The L2PSC_Mask shows MIX1 / MIX2 class pixels below and to the left of the large STS cloud and also in the regions outside of the TTE and $T_{\mathrm{NAT}}$ contours. We also note that although the minimum temperatures along a number of the back trajectories passed below the frost point within 2 days of the MLS/CALIOP observations, no ice PSCs were detected.

Examination of the overlaps between the high TTE values (red contour) and local temperature minima (blue contour) in the cases discussed above reveals a correspondence to $\mathrm{HNO}_{3}$ uptake but a frequent lack of coincident PSC detections. The local temperatures are just as low (and sufficient for STS formation) as in the areas outside the overlaps, but the STS class is not seen at all, whereas the TTE increases substantially. The corresponding detailed time histories of the temperatures at $32 \mathrm{hPa}$ are shown in Fig. 8d and reveal that the remarkable asymmetries in the along-track location of $\mathrm{HNO}_{3}$ uptake with respect to the local temperature minimum distribution can be understood in terms of the different
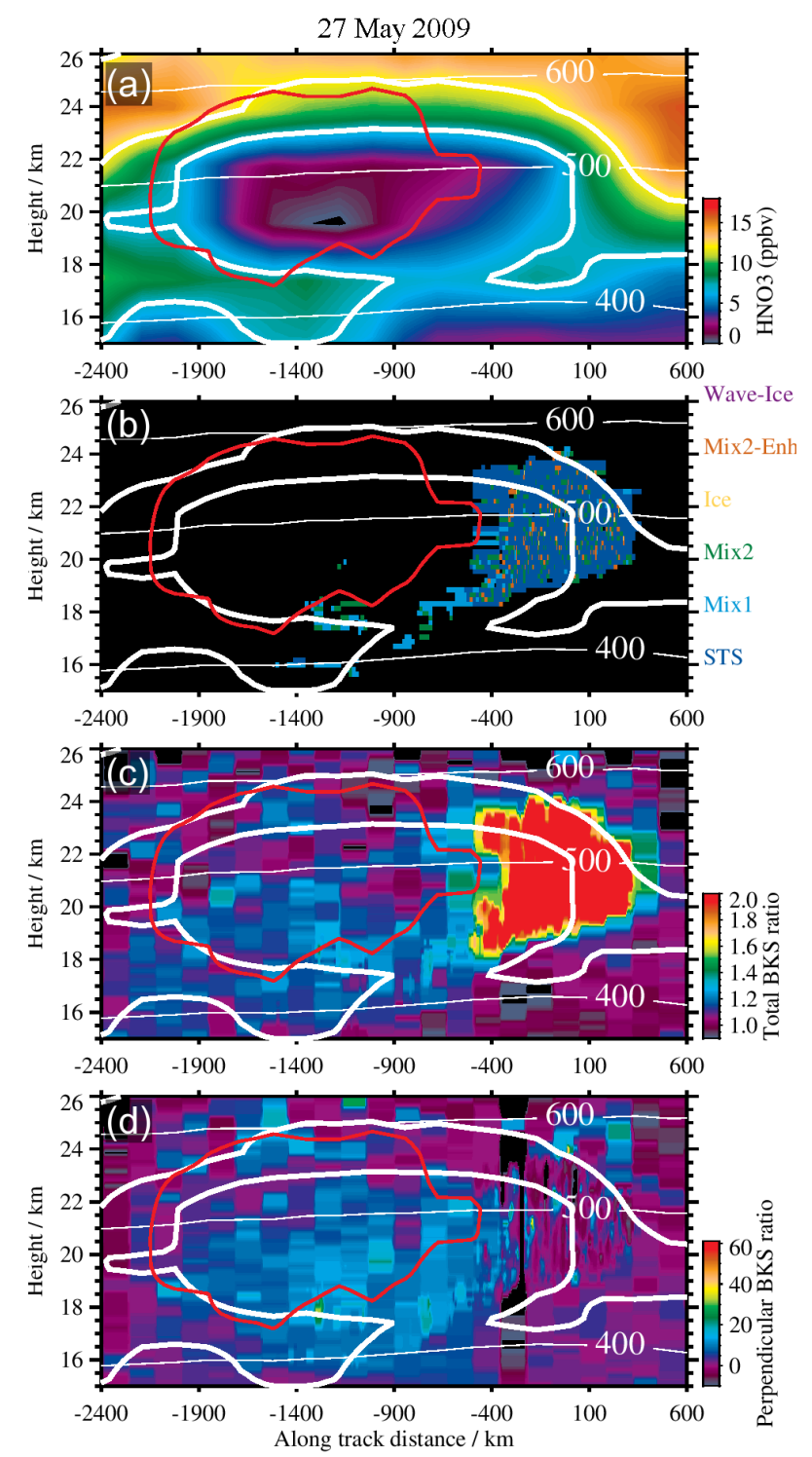

Figure 9. Comparison of along-track data for a partial orbit on 27 May 2009: (a) MLS $\mathrm{HNO}_{3}$ showing sequestration and the central region of total denitrification. (b) CALIOP PSC Mask does not show a corresponding large-scale detection of MIX1 clouds. (c) Smoothed CALIOP total backscatter ratio. (d) Smoothed CALIOP perpendicular backscatter ratio. Solid thick white contours are the $\mathrm{MLS} \mathrm{HNO}_{3}$ isolines for 7 and $12 \mathrm{ppbv} \mathrm{HNO}_{3}$. Red contours indicate TTE values $\geq 3$ days. The detection of solid particle PSCs is evident in the smoothed CALIOP perpendicular backscatter ratio and extends below the TTE contour by $2-4 \mathrm{~km}$.

rates of cooling of particular air parcels. This indicates that in the regions where slow cooling forms NAT first (marked by long white arrows in cases 2 and 4), $\mathrm{HNO}_{3}$ is sequestered into NAT, and therefore STS cannot grow. In contrast, there are regions where fast cooling occurs and STS forms without prior NAT formation (marked by short white arrows in cases 2 and 4). The NAT exists as large particle/low number 
density clouds (sedimenting, sub-visible MIX1) that contain enough condensed $\mathrm{HNO}_{3}$ to be detectable through MLS gasphase depletion, but have low lidar backscatter and are invisible to CALIOP. Alternatively, it could be argued that the NAT particles grew so large further upstream that they were effectively removed to lower altitudes through sedimentation at the time of the orbit crossing observations, leaving behind a permanently denitrified air mass detected by MLS but without coincident CALIOP PSC detections. However, further averaging of CALIOP backscatter (as discussed in Sect. 3) on 27 May, shown in Fig. 9, does indicate a considerably larger area of solid particle detections (MIX1 class), associated with the high TTE region, and so it appears that we are dealing with the limit of the L2PSCMask detection range. Sedimentation of large NAT is suggested by the $2-4 \mathrm{~km}$ region of solid particle detections lying below the high TTE region.

\subsection{Denitrification and renitrification}

The Antarctic gas-phase $\mathrm{HNO}_{3}$ distribution shown in Fig. 10 provides a record of the effects of the formation and dissipation of PSCs and is displayed as the daily areal coverage for equivalent latitudes less than $60^{\circ} \mathrm{S}$, for isentropic levels from 340 to $500 \mathrm{~K}$. The white solid lines indicate low, median, and high values of the $\mathrm{HNO}_{3}$ probability density function. The minimum $\mathrm{HNO}_{3}$ mixing ratios (i.e., the colored region below the 10th percentile white line) indicate that PSCs can lead to a complete removal of the available ambient $\mathrm{HNO}_{3}$ from the gas phase. As shown in Lambert et al. (2012), the spread of $\mathrm{HNO}_{3}$ mixing ratios in totally denitrified regions is compatible with the MLS precision. The temperature decrease starts from the upper levels of the vortex and descends over time, resulting in PSCs and $\mathrm{HNO}_{3}$ uptake developing later at the lower levels. The maximum $\mathrm{HNO}_{3}$ mixing ratios (i.e., the colored region above the 90th percentile white line) indicate episodes of renitrification at the lower levels arising from the sedimentation of PSCs. As the NAT particles fall through lower levels, they may pass into regions where they are less thermodynamically stable. In these cases the evaporative release of $\mathrm{HNO}_{3}$ from the condensed phase increases the gas-phase values, and this is detected as a rise in the $\mathrm{HNO}_{3}$ measured by MLS at the lower levels. The process is seen quite clearly in the increasing time lag between the appearance of anomalously large $\mathrm{HNO}_{3}$ values above the 90th percentile at $420 \mathrm{~K}$ (mid-May) compared to $340 \mathrm{~K}$ (midJune). This process acts to raise the NAT temperature existence threshold at the lower levels because of the enhanced gas-phase $\mathrm{HNO}_{3}$ and therefore increases the likelihood of occurrence of NAT. Heterogeneous chemical reactions on the PSC particles involving $\mathrm{ClONO}_{2}$ and $\mathrm{N}_{2} \mathrm{O}_{5}$ produce additional $\mathrm{HNO}_{3}$, which remains in the condensed phase until the PSC dissipates (Turco et al., 1989). Therefore, an increase in gas-phase $\mathrm{HNO}_{3}$ at a given level may arise from the evaporation either of sedimenting PSCs from above or

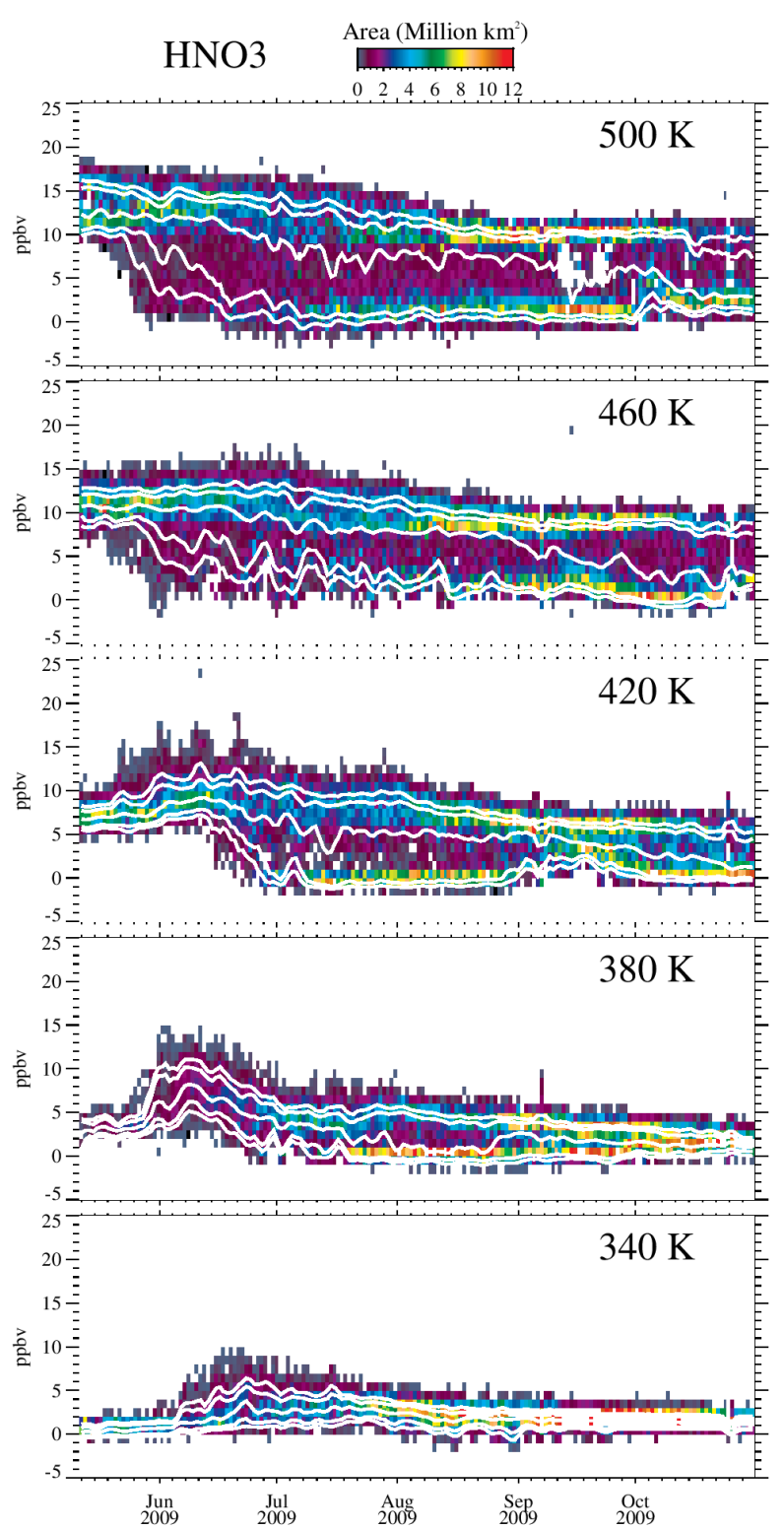

Figure 10. Time series of the distribution of $\mathrm{MLS} \mathrm{HNO}_{3}$ in the Antarctic from May to October 2009, for equivalent latitudes less than $60^{\circ} \mathrm{S}$, and for isentropic levels from 340 to $500 \mathrm{~K}$. Major tick marks indicate the beginning of the month. The color scale indicates the areal coverage. White solid lines indicate the 10th, 20th, 50th (i.e., median), 80th, and 90th percentiles of the $\mathrm{HNO}_{3}$ probability density function.

from extant PSCs that release the excess $\mathrm{HNO}_{3}$ formed as a product of heterogeneous reactions. As the Antarctic winter progresses and the temperatures at the lower levels decrease, the redistributed $\mathrm{HNO}_{3}$ is itself subject to further uptake into STS/NAT, resulting in potential further denitrification and consequent removal of $\mathrm{HNO}_{3}$ from a larger vertical range of the lower stratosphere. 
(a)

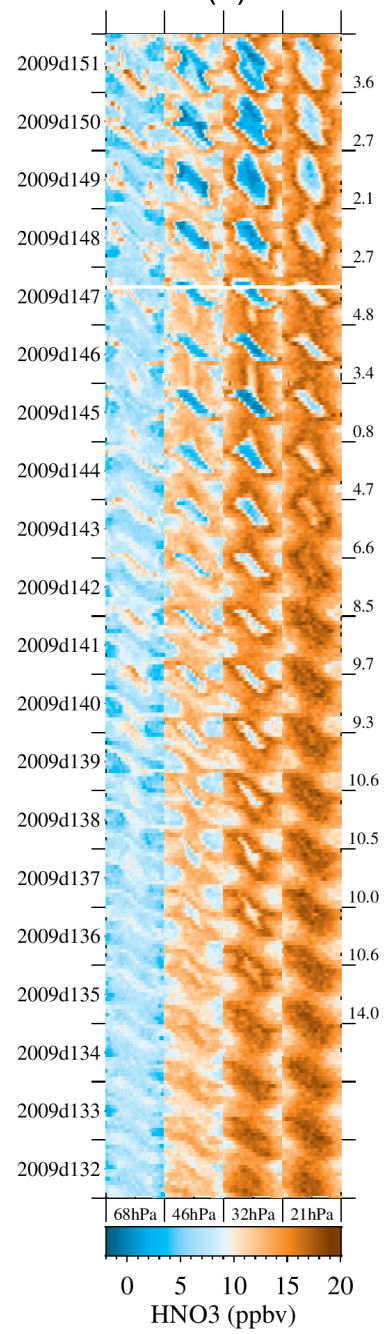

(b)

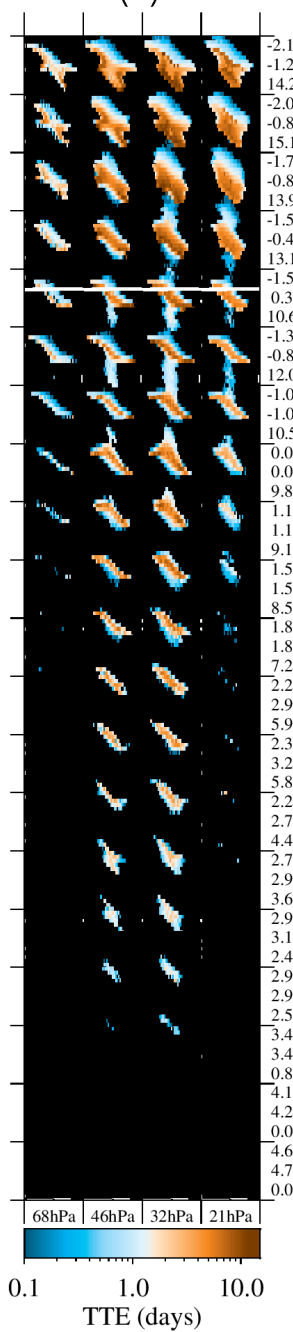

(c)

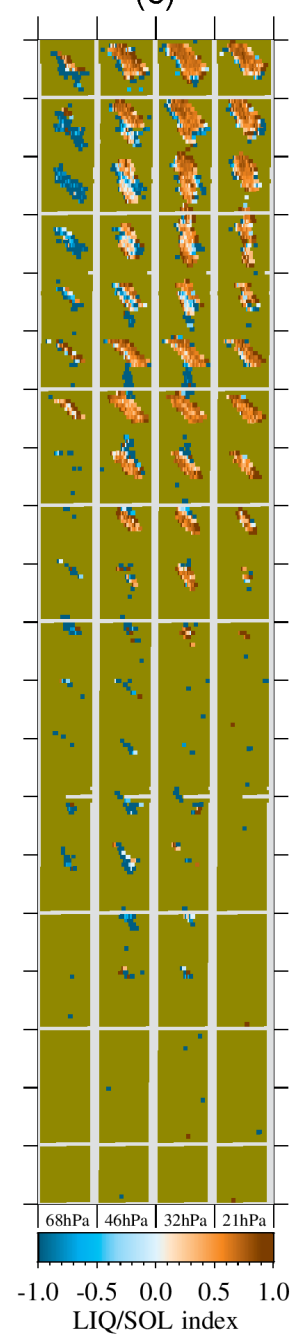

(d)

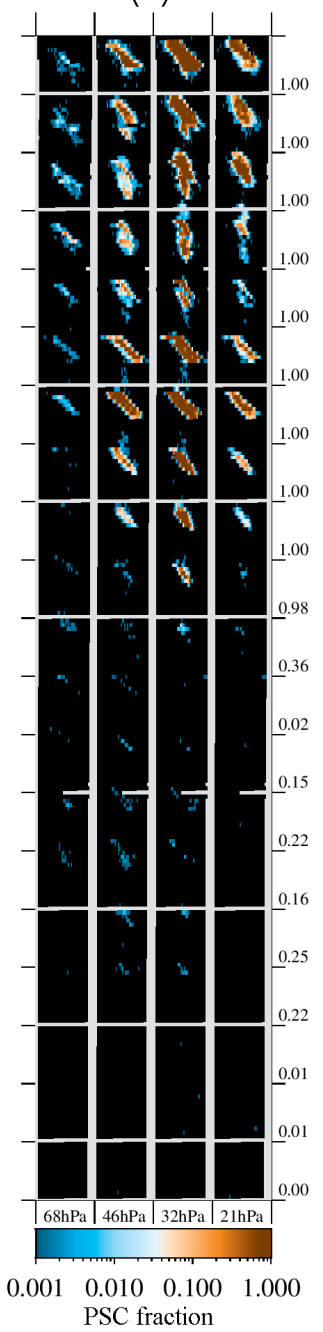

Figure 11. Twenty-day time series of Antarctic raster plots at 68, 46, 32, and $21 \mathrm{hPa}$ in 2009 from day number 132 (12 May) to 151 (31 May). Gray shading indicates no observations; olive-green shading indicates observations but no detections. (a) $\mathrm{MLS} \mathrm{HNO}_{3}$. Numbers on the right axis indicate the median $\mathrm{HNO}_{3}$ for the region where TTE $>2$ days (in ppbv) for each day at $32 \mathrm{hPa}$. (b) Temperature history. A set of three numbers on the right axis for each day at $32 \mathrm{hPa}$ indicates in ascending order (i) maximum TTE in days, (ii) minimum local temperature, $T-T_{\text {ICE }}$ in K, (iii) minimum temperature encountered along the back-trajectory, and $T-T_{\text {ICE }}$ in K. (c) CALIOP LS_index. (d) CALIOP PSC fraction. Numbers on the right axis indicate the maximum PSC fraction (ratio of the number of PSC detections to the number of observations) for each day at $32 \mathrm{hPa}$.

\section{Time series of PSC formation and Lagrangian temperature history}

In this section we examine the formation of PSCs and the Lagrangian temperature history during the early 2009 Antarctic PSC season. Figure 11 shows a time series of data taken over the 20 days from 2009d132 to 2009d151 (12-31 May) of $\mathrm{HNO}_{3}$, TTE (temperature history), LS_index, and PSC fraction. Averaging bins are as described in Sect. 4.1. There are four columns in each panel corresponding to four pressure levels at 68, 46, 32, and $21 \mathrm{hPa}$. Each "mini-plot" (e.g., identified by $\mathrm{HNO}_{3} 2009 \mathrm{~d} 13268 \mathrm{hPa}$ ) is a raster image with the axes shown in Fig. 2 (the reduction in plot size merges together the square pixels; there is no longer any visible white space between the measurement locations). The first column $\left(68 \mathrm{hPa}\right.$ ) for $\mathrm{HNO}_{3}$ consists of 20 such images, one for each day stacked one above the other. The second column is plotted adjacent and corresponds to $\mathrm{HNO}_{3}$ at $46 \mathrm{hPa}$ and so on for the third $(32 \mathrm{hPa})$ and fourth $(21 \mathrm{hPa})$ columns. TTE is the Lagrangian temperature threshold exposure as discussed in connection with Fig. 8. The LS_index is as described before for each averaging bin (gray shading indicates no data, e.g., instrument off or daytime CALIOP observations; olivegreen shading indicates operations but no detections). PSC 
fraction is the ratio of the number of PSC detections to the total number of observations in each averaging bin.

Significant TTE first appears on the $32 \mathrm{hPa}$ level close to the South Pole on 2009d134/2009d135 (14-15 May), gradually increases in area, and expands in vertical extent rapidly to the $46 \mathrm{hPa}$ level and eventually to the 68 and $21 \mathrm{hPa}$ levels with a delay of about a week. Note the lack of corresponding areal coverage of PSCs, especially on the $32 \mathrm{hPa}$ level (only a few PSC detections are scattered about; see the PSC fraction panel). The PSC LS_index indicates some predominantly solid class PSC detections at 68 and $46 \mathrm{hPa}$ before 2009d141 (21 May), but there are fewer detections at $32 \mathrm{hPa}$. However, MLS indicates $\mathrm{HNO}_{3}$ uptake on the $32 \mathrm{hPa}$ level starting earlier from $2009 \mathrm{~d} 135$ (15 May) that is as widespread as that on the $46 \mathrm{hPa}$ level and similar in areal extent to the TTE. At $68 \mathrm{hPa}$ there is evidence for an increase in $\mathrm{HNO}_{3}$ following 2009d139 (19 May), presumably due to renitrification from evaporating NAT PSCs that have sedimented from a higher level. The combined data at $32 \mathrm{hPa}$ indicate that, in the early period, the CALIOP L2PSCMask is not detecting PSCs, since there is a persistent area of daily $\mathrm{HNO}_{3}$ depletion that is not matched by corresponding PSC detections but is consistent with the temperature history. We use the term subvisible PSCs to refer to these cases (i.e., significant depletion of gas-phase $\mathrm{HNO}_{3}$, but without detection by CALIOP).

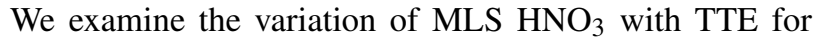
sub-visible PSCs in the period 2009d135-2009d144 (1524 May) in more detail in Fig. 12. The scatter plot of the gas-phase $\mathrm{HNO}_{3}$ values shows a range of $\sim 4 \mathrm{ppbv}$ (from $\sim 14$ to $\sim 18 \mathrm{ppbv}$ ) for low TTE, and as TTE increases beyond $\sim 1$ day, the $\mathrm{HNO}_{3}$ decreases. A highly simplified microphysical model has been used to calculate the uptake of $\mathrm{HNO}_{3}$ from the gas phase. Growth of NAT by vapor deposition has been calculated in the manner outlined by Toon et al. (1989) and following the simplifications introduced by Carslaw et al. (2002). Here, we ignore sedimentation and calculate the growth of monodisperse NAT in a constant temperature atmosphere $\left(T_{\mathrm{ICE}}+4 \mathrm{~K}\right)$ for two different initial NAT densities $\left(5 \times 10^{-4}\right.$ and $\left.5 \times 10^{-5} \mathrm{~cm}^{-3}\right)$ and two initial total $\mathrm{HNO}_{3}$ values that encompass the observed range (14 and $18 \mathrm{ppbv}$ ). The NAT growth model is initialized with a $0.1 \mu \mathrm{m}$ radius at the nucleation time $(\mathrm{TTE}=0)$, and the subsequent time evolution of the gas-phase $\mathrm{HNO}_{3}$ is plotted as colored curves indicating the NAT particle radius. These two curves practically bound the MLS observations of the distribution of gas-phase $\mathrm{HNO}_{3}$ as a function of TTE. As is well known, low NAT number densities produce large NAT particles (Jensen et al., 2002), which take several days to reach thermodynamic equilibrium (the uptake curves bottom out after 15 days or more). Backscatter calculations for NAT with characteristics of the upper curve (assuming $\epsilon=0.9$ ) show that the backscatter (perpendicular or total) is below the CALIOP threshold of detection along the entire time evolution. For the lower curve, the higher NAT number density limits the particle growth to a much smaller radius, and backscatter

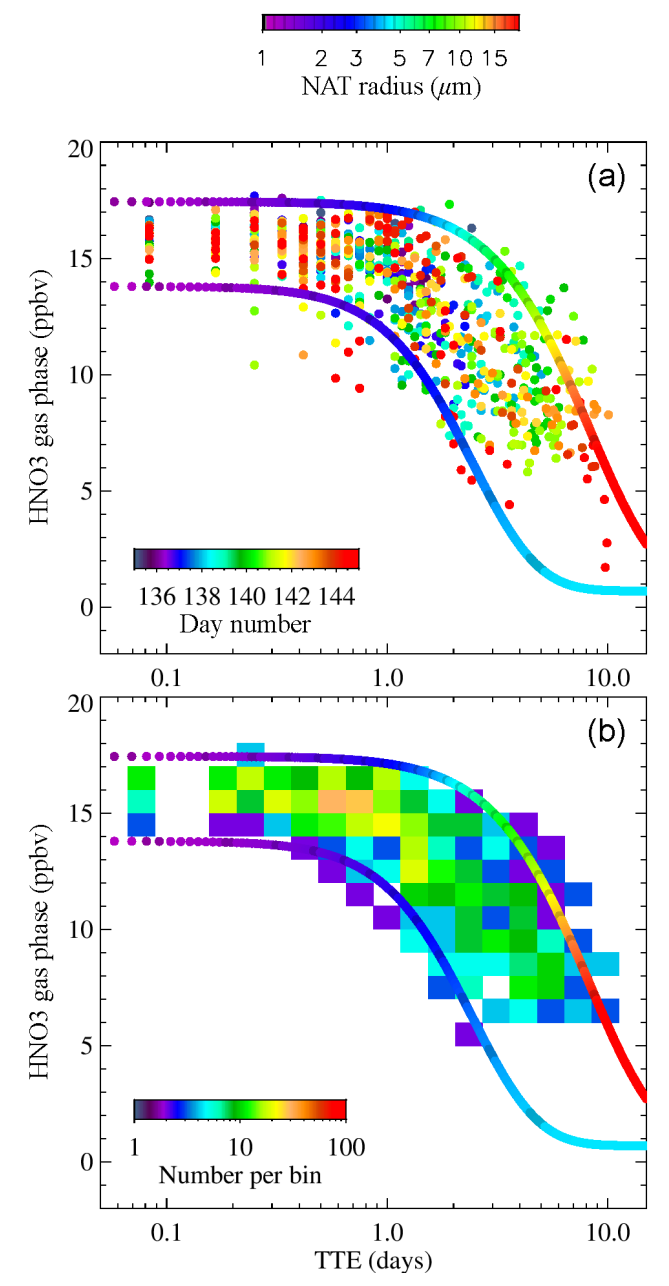

Figure 12. $\mathrm{MLS} \mathrm{HNO}_{3}$ on the $32 \mathrm{hPa}$ pressure level over the 10-day period from 15 to 24 May 2009 (days 135-144) in the Antarctic polar vortex. Data are selected only if there are no coincident PSC detections by CALIOP. (a) Scatter plot of individual $\mathrm{HNO}_{3}$ values vs. TTE. The colored dots indicate the measurement day number (given in the inset color bar). The two colored curves are from a calculation of the gas-phase $\mathrm{HNO}_{3}$, assuming growth of NAT at a constant temperature of $T_{\mathrm{ICE}}+4 \mathrm{~K}$ for NAT number densities and initial total $\mathrm{HNO}_{3}$ values of $5 \times 10^{-5} \mathrm{~cm}^{-3}$ and $18 \mathrm{ppbv}$, respectively, for the upper curve and $5 \times 10^{-4} \mathrm{~cm}^{-3}$ and $14 \mathrm{ppbv}$ for the lower curve. The top color bar indicates the NAT radius. (b) As in (a) except plotted as a 2-D histogram of the density of points, with the inset color bar giving the number of samples per bin. Only bins accumulating two or more data points are shown.

calculations suggest that the NAT particles should be detectable after 0.8 days following nucleation for the perpendicular backscatter threshold and after 1.3 days for the total backscatter threshold. The details of the scattering calculations depend on assumptions about the underlying particle characteristics, for which we lack definitive knowledge. However, observationally we repeatedly see large depletions of $\mathrm{HNO}_{3}$ without accompanying particle detections, which 


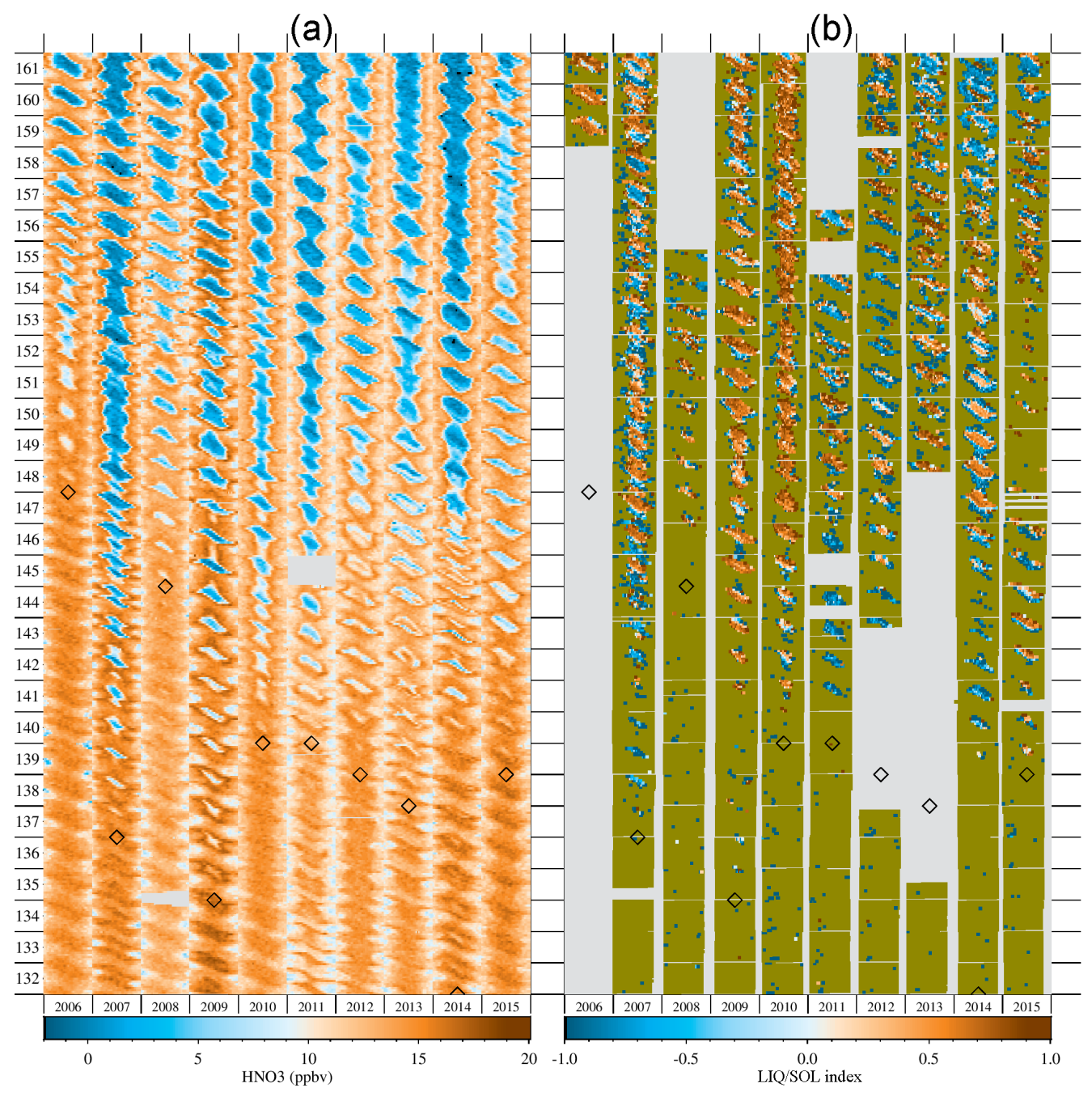

Figure 13. Thirty-day time series of Antarctic raster plots at $32 \mathrm{hPa}$ for 2006-2015. Diamond symbols indicate the last day before the start of detectable $\mathrm{HNO}_{3}$ depletion. Gray shading indicates no observations; olive-green shading indicates observations but no detections. Day number is shown on the vertical axis. (a) MLS gas-phase $\mathrm{HNO}_{3}$. (b) CALIOP LS_index.

can be accounted for qualitatively by a NAT population characterized by low number densities/large radii.

\section{Interannual variations in the early Antarctic PSC season}

In this section we investigate the interannual variability of Antarctic PSCs and $\mathrm{HNO}_{3}$ over the past decade and again make use of the geolocated raster plot format discussed in Sect. 2.4. Figure 13 shows the MLS $\mathrm{HNO}_{3}$ and CALIOP LS_index at $32 \mathrm{hPa}$ for 30 days from day number 132 to 161 (12 May to 10 June) for the years 2006-2015. The corresponding time series for TTE and $T-T_{\text {ICE }}$ are shown in Fig. 14. Additionally, in Fig. 15, we present a complementary side-by-side comparison of the same observations by plotting the $\mathrm{MLS} \mathrm{HNO}_{3}$ as a time series with each observa- tion colored according to the TTE (Fig. 15a) or the CALIOP LS_index (Fig. 15b). The evolution of the Antarctic dataset of $\mathrm{HNO}_{3}$, LS_index, and the corresponding TTE and $T-$ $T_{\text {ICE }}$ can then be followed as a function of time by reference to the above figures. In this analysis we have not treated ice PSCs separately and they are counted as part of the solid PSC population. However, in general, the number of PSCs classified as ice types in the time period considered here is low and constitutes less than $2 \%$ of the total PSC detections, except for the years 2007 and 2011, in which ice is $6 \%$ of the total and accompanying dehydration is observed about a week before the end of the time period. The extent to which the polar vortex distorts and wanders around the South Pole can be assessed by a dynamical diagnostic such as the vortextemperature concentricity (VTC). This hybrid temperaturevortex diagnostic (Lawrence et al., 2015, see their Eq. 1 and Fig. 16) indicates that, for the Southern Hemisphere, the cold 


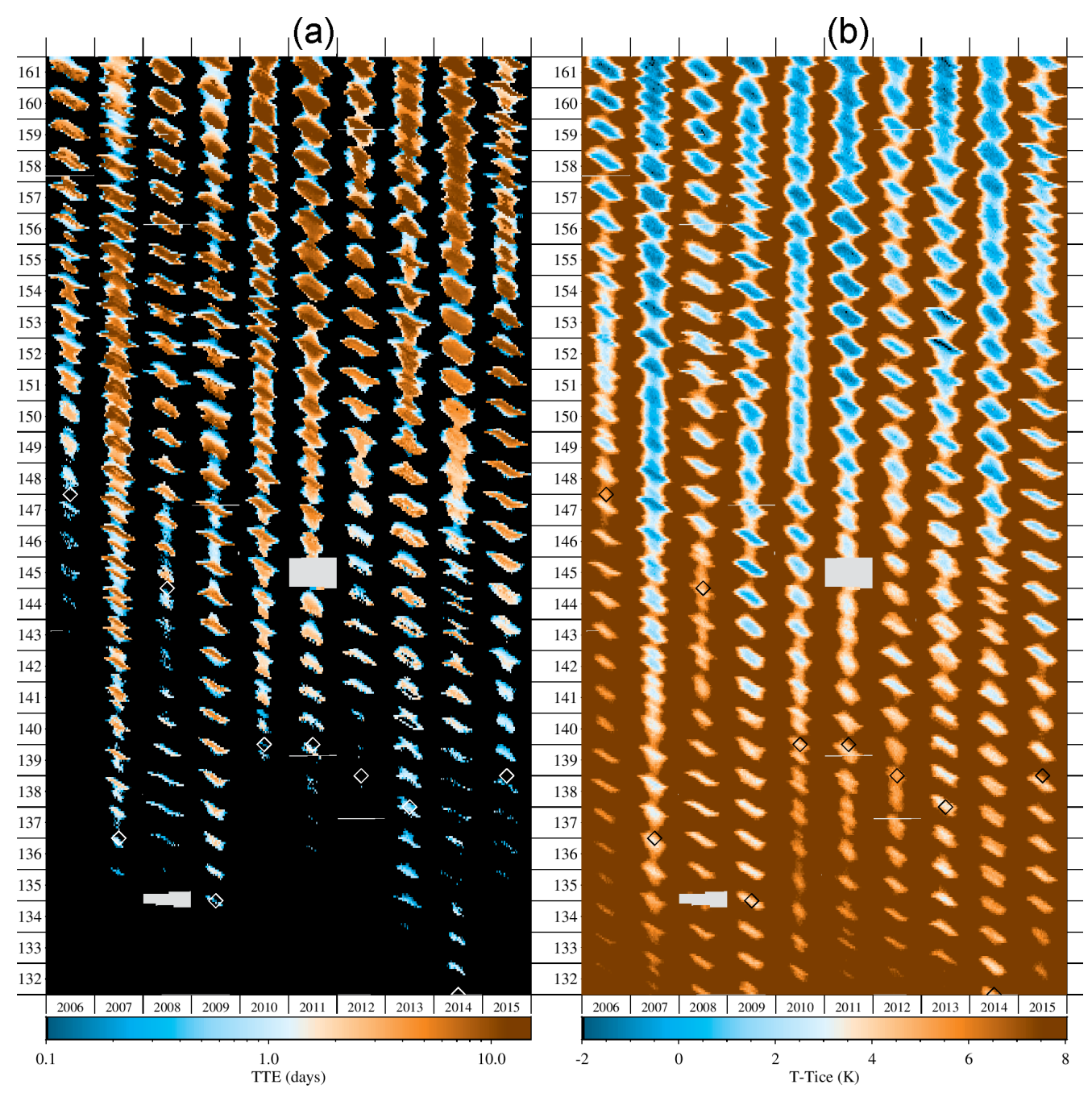

Figure 14. Thirty-day time series of Antarctic raster plots at $32 \mathrm{hPa}$ for 2006-2015. Day number is shown on the vertical axis. (a) TTE (days). (b) $T-T_{\mathrm{ICE}}(\mathrm{K})$.

pool temperatures and the polar vortex are highly concentric. We have also investigated the daily scatter of temperature vs. vorticity in the ERA-Interim reanalysis data (not shown here), and find that the annular region 60 to $82^{\circ} \mathrm{S}$ (visible to Aura and CALIOP) describes adequately the state of the region poleward of $82^{\circ} \mathrm{S}$ (not sampled by Aura or CALIOP).

The start of the PSC season appears to display two modes (see Figs. 13b and 15b), with some years having larger coverage of solid PSCs $(2007,2011$, and 2014) than others, which show larger coverage of liquid PSCs $(2008,2009,2010$, and 2015). We identify the presence of sub-visible PSCs by a delay between the onset of gas-phase $\mathrm{HNO}_{3}$ depletion (see Figs. 13a and 15a) and the geophysically associated detection of PSCs (see Figs. 13b and 15b). Table 2 lists the day number of the initial onset of $\mathrm{HNO}_{3}$ depletion, the first day of detection of PSCs by CALIOP, the presence of sub-visible PSCs, and the predominant PSC class (solid or liquid) for the first few days following detection. Sub-visible clouds are present in 5 out of the 7 years with MLS and CALIOP overlap during the critical time period when uptake of gasphase $\mathrm{HNO}_{3}$ is first detected. The time lag between the observed depletion of gas-phase $\mathrm{HNO}_{3}$ and the detection of PSCs varies and is largest in 2009 and 2014. We infer that the sub-visible PSCs are composed of NAT because the expected uptake of $\mathrm{HNO}_{3}$ is negligible for STS at the associated ambient temperatures (see Fig. 14b). In 2015 there is a notable period of several days (148-153) without many PSC detections (see Figs. $13 \mathrm{~b}$ and $15 \mathrm{~b}$ ), although $\mathrm{HNO}_{3}$ is still consistently low (see Fig. 13a), probably indicating sedimentation of NAT and permanent denitrification. However, since Woiwode et al. (2014) have reported the presence of NAT with larger apparent sizes compared to compact spherical particles and with concomitant reduced settling rates, we must also consider the possibility that the NAT radius grew larger and subsequently evolved into sub-visible NAT. 
Table 2. Comparison of times of occurrence of $\mathrm{HNO}_{3}$ depletion and detection of PSCs at the start of each PSC season.

\begin{tabular}{rrrll}
\hline Year & Onset day of $\mathrm{HNO}_{3}$ depletion & First day of PSC detection & Sub-visible PSCs & Dominant PSC class \\
\hline 2006 & 148 & $*$ & $*$ & $*$ \\
2007 & 137 & 137 & No & SOL \\
2008 & 145 & 147 & Yes & LIQ \\
2009 & 135 & 141 & Yes & LIQ \\
2010 & 140 & 141 & Yes & LIQ \\
2011 & 140 & 141 & Yes & SOL \\
2012 & 139 & $*$ & $*$ & $*$ \\
2013 & 138 & $*$ & $*$ & $*$ \\
2014 & 133 & 139 & Yes & SOL \\
2015 & 139 & 139 & No & LIQ \\
\hline
\end{tabular}

* indicates no MLS and CALIOP overlap.

Höpfner et al. (2009) and Spang et al. (2016) presented intercomparisons of CALIOP vs. MIPAS PSC detections and statistics on the frequency of type classifications that are relevant here. During May, considerably more MIPAS-only PSC detections (i.e., without corresponding matching detections by CALIOP) were found by Höpfner et al. (2009), and even with longer-term averaging, over the entire May-October period, Spang et al. (2016) concluded that MIPAS has higher sensitivity to PSCs since, for the cases where CALIOP detects no cloud, about $60 \%$ correspond to cloudy scenes for MIPAS. Therefore, these MIPAS/CALIOP comparisons support our conclusion that CALIOP is not sensitive to low number density and large particle NAT.

The goal of describing the interannual variability of PSC seasons within the context of a decadal climatology can be met by synthesizing the CALIOP and MLS observations, consisting of the four-variable dataset $\left(\mathrm{HNO}_{3}\right.$, TTE, $T-T_{\mathrm{ICE}}$ and LS_index), into a number of characteristic groups or clusters and thereby capturing the major characteristics of PSC formation. Simple partitioning obtained by setting a single threshold boundary on each of the four variables would result in 16 different PSC groups (some of which may be empty). Such a large number of groupings would complicate interpretation, and so a more parsimonious scheme is desirable to enforce a reduction in the number of groups. Cluster analysis (CA) is a well-established tool for unsupervised exploration of a dataset (Jain, 2010). Ideally members of the same cluster (identified by their proximity to the cluster centroid) will naturally exhibit similar intrinsic properties and display low within-cluster variance, whereas the differences amongst the members of one cluster to another will display high between-cluster variance. We used a $K$ means centroid algorithm with a Euclidian distance metric to partition the four-variable dataset after performing zero mean and unit variance normalization. $K$ means analyses were performed for a varying number of imposed clusters ( $K=2$ to $K=20$ ), and the gap statistic (Tibshirani et al., 2001) was used to assess the optimal number of clusters by comparison with uni- formly random data and an uncorrelated dataset obtained by randomly reordering the measurement times of the four variables. A steep rise in the gap statistic was observed over the range $K=2$ to $K=7$, followed by a flattened plateau region out to $K=20$ (not shown). The clusterings obtained for $K=6$ are shown in Table 3 and Fig. 16 for all the years 2006-2015. The $K=6$ case was chosen because the clusters fell practically into two sets $(\mathrm{a}, \mathrm{b})$ of three groups, corresponding to predominantly liquid (Fig. 16a, LS_index $>0$ ) and predominantly solid (Fig. 16b, LS_index $<0$ ) PSCs. No particular improvement was observed for the $K=7$ case. The LS_index vs. day number scatter plot indicates that there is some cross-over of liquid/solid PSCs present in both the a1 and a2 groups. The groups are presented superposed as a scatter plot on the thermodynamic $\mathrm{HNO}_{3}$ vs. $T-T_{\mathrm{ICE}}$ diagram and show clearly that the two sets $(a, b)$ are associated with the STS and NAT equilibrium branches. As in previous analyses, the NAT population exhibits considerable nonequilibrium effects (Lambert et al., 2012; Pitts et al., 2013), with the temperature distribution extending to temperatures as low as the STS branch. No correction has been made for the suspected temperature bias in the meteorological temperature data (Sect. 3.2).

The stereographic map projections in Fig. 16 show the PSC groups with higher temperatures (a3, b2, b3) extending to lower latitudes, reflecting the general outward radial increase in the polar temperature distribution. However, in particular, group b3 displays similarities to a circumpolar NAT belt (Tabazadeh et al., 2001; Höpfner et al., 2006a), although it is notable that this group also includes CALIOP detections at temperatures apparently higher than the NAT existence threshold $T_{\mathrm{NAT}}=T_{\mathrm{ICE}}+7 \mathrm{~K}$. The b3 group temperature history $\left(\mathrm{HNO}_{3}\right.$ vs. TTE) also shows little exposure to low temperatures. These may result from the inability of the gridded synoptic reanalysis data to capture accurately the local temperature minima. Another possibility is that the NAT particles may survive for a time before fully melting as they 
(a) MLS

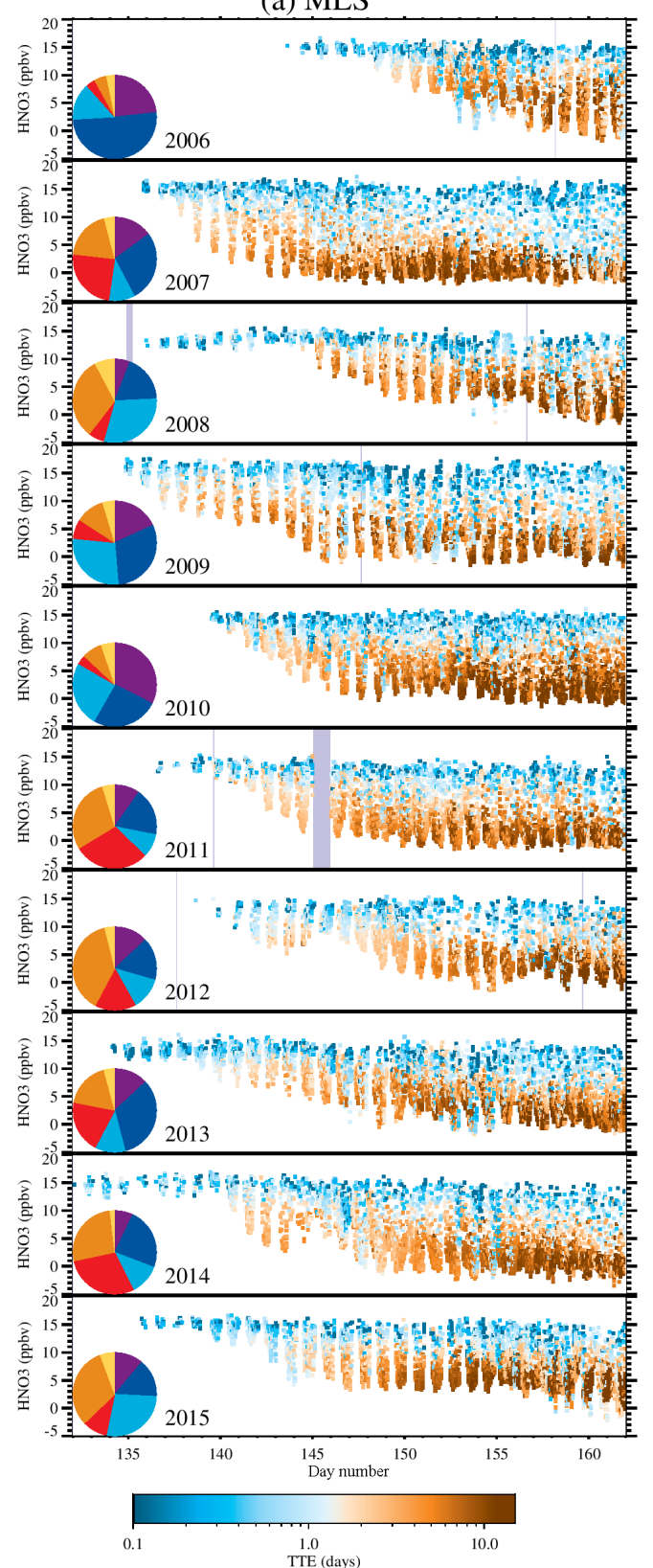

(b) CALIOP

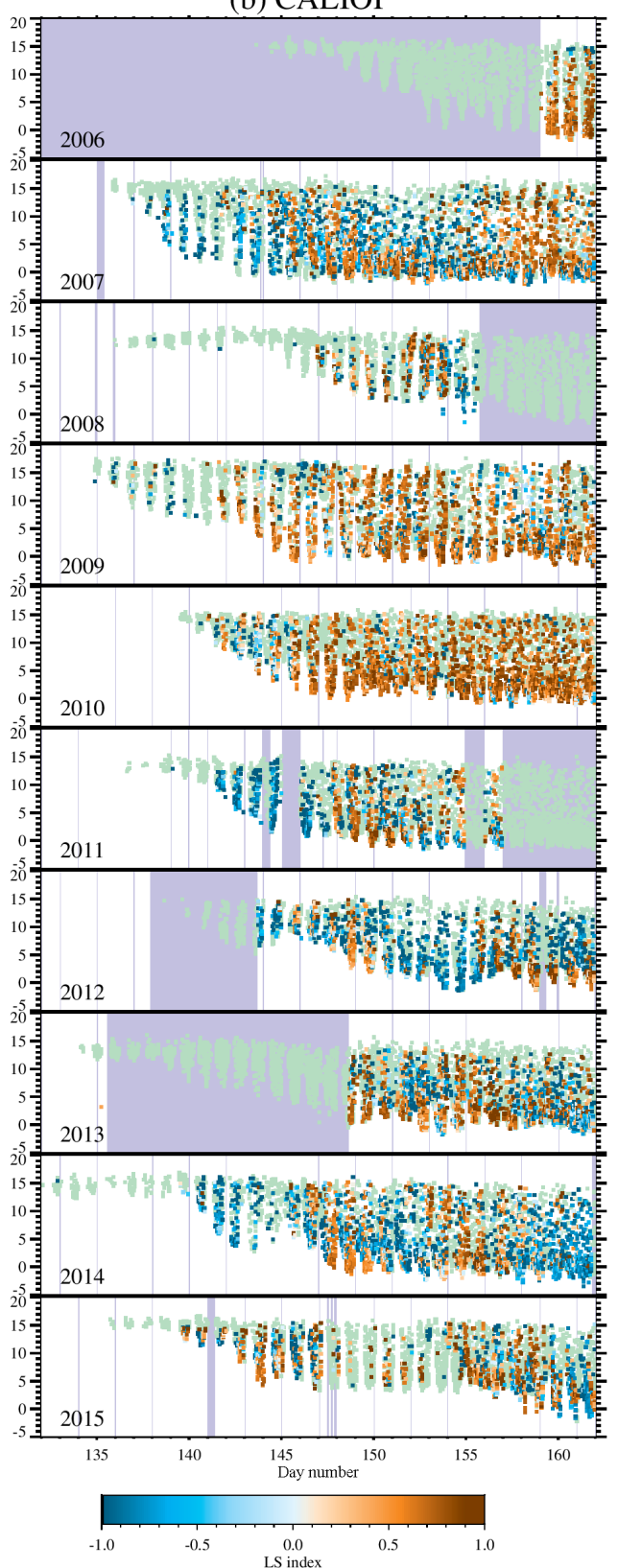

Figure 15. Time series for the Antarctic for associated $\mathrm{HNO}_{3}$ and PSCs during 2006-2015 at $32 \mathrm{hPa}$. (a) MLS HNO 3 vs. day number. The color scale shows the TTE. Only data for TTE $>0.1$ day are plotted. The inset pie charts indicate the relative proportions of measurements in six categories (red-yellow sectors are solid PSCs; purple-blue sectors are liquid PSCs) as defined in the text and in Fig. 16. (b) CALIOP LS_index vs. day number. The color scale shows the LS_index. Gray shading indicates no measurements. Light green shading in (b) indicates the envelope of $\mathrm{MLS}_{\mathrm{HNO}}$ observations in (a).

are advected downstream (Lambert et al., 2012) away from their gravity-wave sites of origin.

Compelling evidence of the differences in the formation of liquid and solid PSCs can be deduced from comparisons of the temperature history of the groups. For the case of moderate gas-phase $\mathrm{HNO}_{3}$ depletion, the occurrence of liquid PSCs (a3) shows a steep fall-off in the TTE distribution beyond $\sim 1$ day. In contrast, the occurrence of solid PSCs (b2) extends to TTE $\sim$ several days and also shows a trend of declining $\mathrm{HNO}_{3}$ with increasing TTE that can be explained by reference to the NAT growth curves modeled in Fig. 12. For the case of large gas-phase $\mathrm{HNO}_{3}$ depletion, the b1 group shows a peak around TTE $=6$ days, and very few of these solid PSCs formed at TTE $<2$ days. This is also a conse- 
(a)
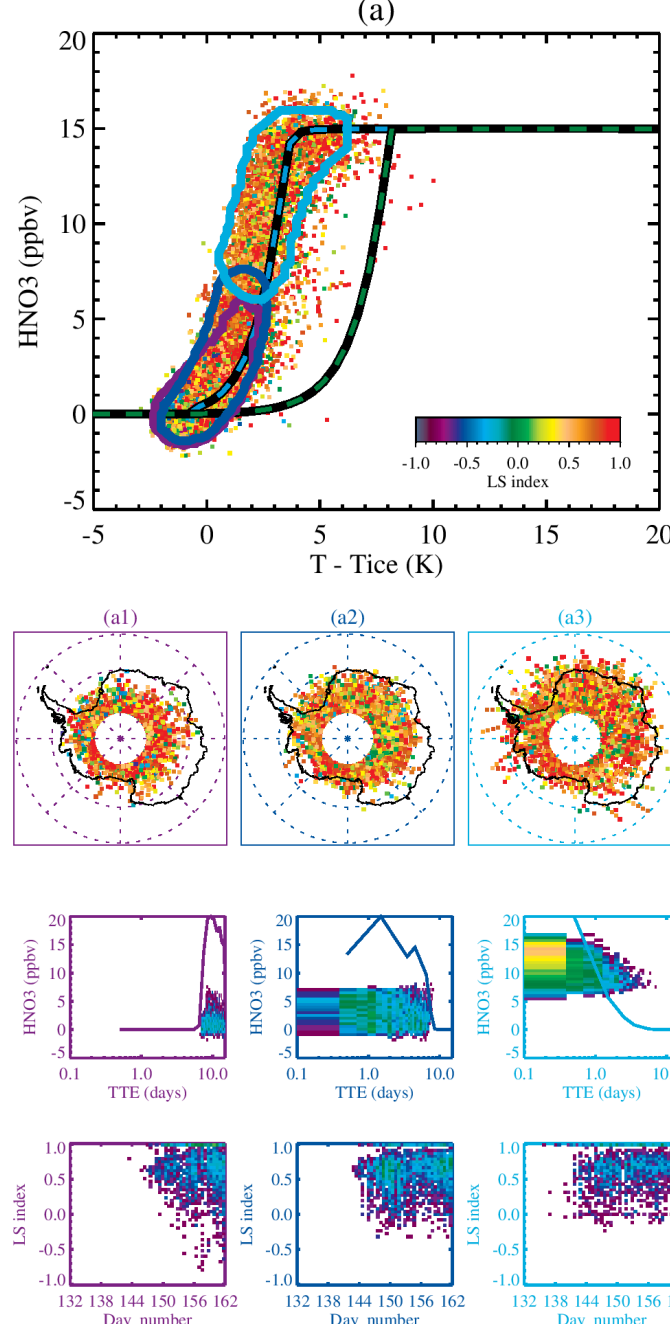

(a2)
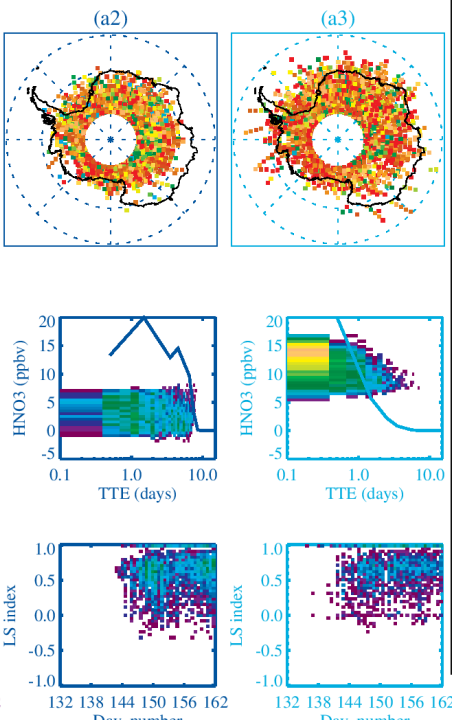

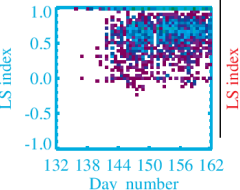

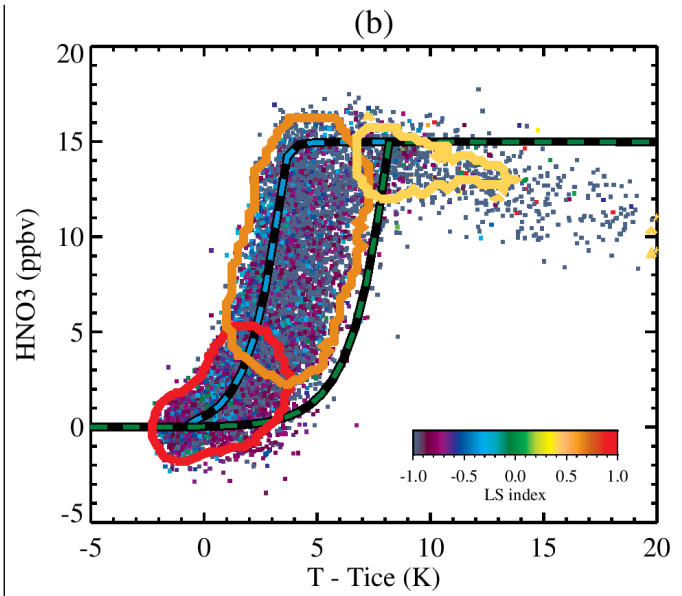
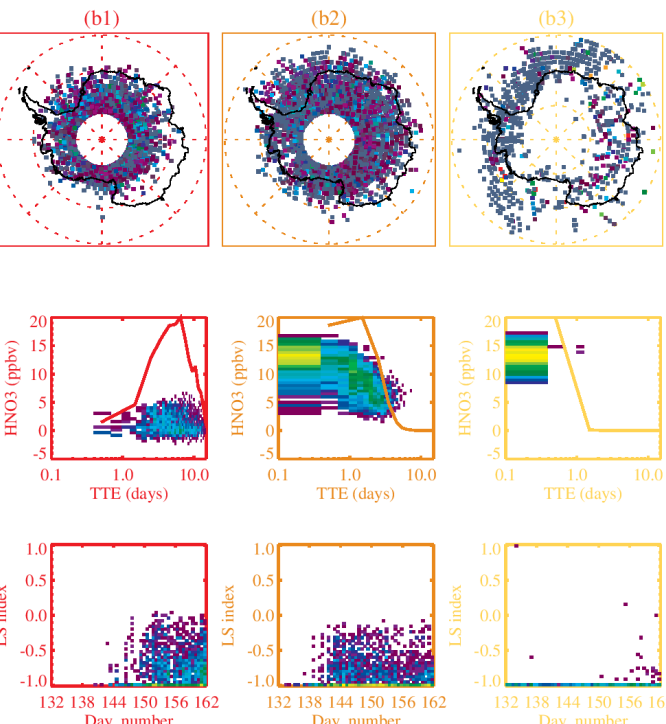

Figure 16. Results of the $K=6$ cluster analysis of the combined MLS and CALIOP data for the Antarctic for the years 2006-2015 at $32 \mathrm{hPa}$. Thermodynamic diagrams indicate for reference the theoretical $\mathrm{HNO}_{3}$ uptake by STS (blue-black dashed line) and by NAT (green-black

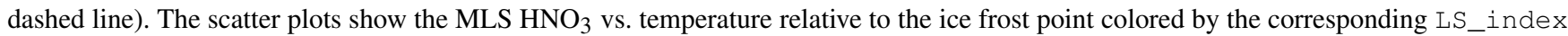
obtained from CALIOP. Column a (b) shows the data in three groups a1-a3 (b1-b3), which are seen to be predominantly liquid (solid) PSC detections according to the LS_index. The colored contours ( $\mathbf{a} 1$ - purple, a2 - dark blue, a3 - light blue, b1 - red, b2 - orange, b3 - yellow) contain $95 \%$ of the corresponding cluster members. The maps show the geographic distribution of the members of each of the clusters and are color-coded by the LS_index (inset color bar). A 2-D view $\left(\mathrm{HNO}_{3}\right.$ vs. TTE) is provided of the cluster densities (purpleyellow shading indicates low to high density) and the accompanying colored solid lines represent the normalized 1-D histogram of the TTE values. The cluster densities are also displayed as a 2-D view of LS_index vs. day number.

Table 3. Results of the $K=6$ cluster analysis for 2006-2015: the number of members assigned to each group, the mean and standard deviation of the relative number of observations, and the centroids in the four-variable data space are given.

\begin{tabular}{|c|c|c|c|c|c|c|c|c|}
\hline $\begin{array}{l}\text { Group } \\
\text { name }\end{array}$ & $\mathrm{LIQ} / \mathrm{SOL}$ & Number & $\begin{array}{r}\text { Mean } \\
(\%)\end{array}$ & $\begin{array}{r}\text { Standard } \\
\text { deviation }(\%)\end{array}$ & $\begin{array}{l}\mathrm{HNO}_{3} \\
\text { (ppbv) }\end{array}$ & $\begin{array}{r}\text { TTE } \\
\text { (days) }\end{array}$ & $\begin{array}{r}T-T_{\text {ICE }} \\
\text { (K) }\end{array}$ & LS_index \\
\hline a1 & LIQ & 3194 & 14 & 8 & 1.41 & 11.22 & 0.07 & 0.56 \\
\hline $\mathrm{a} 2$ & LIQ & 5139 & 23 & 7 & 2.84 & 3.30 & 0.67 & 0.55 \\
\hline a3 & LIQ & 3532 & 18 & 9 & 11.19 & 0.88 & 3.10 & 0.60 \\
\hline b1 & SOL & 3194 & 16 & 10 & 1.33 & 6.80 & 0.71 & -0.73 \\
\hline b2 & SOL & 4206 & 24 & 10 & 9.04 & 1.61 & 4.00 & -0.83 \\
\hline b3 & SOL & 866 & 5 & 1 & 13.25 & 0.02 & 10.97 & -0.90 \\
\hline
\end{tabular}


quence of the microphysics of NAT growth since it requires $\sim$ days for the NAT particles to grow sufficiently to substantially deplete the gas-phase $\mathrm{HNO}_{3}$. Permanent denitrification caused by sedimentation of large NAT is the likely cause of the fall-off in the occurrence of PSCs in the b1 group at large TTE. In contrast, there exists a substantial occurrence of liquid PSCs at all values of TTE in groups a1 and a2, as expected since the growth/evaporation of STS is a faster process dependent on the instantaneous temperature.

The total number of coincident MLS and CALIOP observations over the 10-year time span is $\sim 2 \times 10^{4}$, and the mean (standard deviation) of the relative number of observations (in percent) for group a is 55 (8), and for group b it is 45 (11). Therefore liquid PSCs (group a) are the dominant form and solid PSCs (group b) have slightly more variability. The corresponding breakdown of the mean and standard deviation for the individual groups is given in Table 3.

The observations in each cluster obtained from the decadal climatology were sub-divided to produce data for the individual PSC seasons. The results are shown in the pie charts in Fig. 15 to provide a convenient summary of the interannual variations. Note that the year 2006 cannot be compared to the others because the sampling consists of only 3 days at the end of the season. The pie charts show that the years 2009 and 2010 stand out as the ones with the highest occurrence of liquid PSCs (purple-blue sectors) (77 and 83\%, respectively) in the 10-year record. The remaining years fall into two broadly related categories with a slightly larger proportion of liquid PSCs in 2007 (52\%), 2008 (54\%), 2013 $(58 \%)$, and $2015(53 \%)$ and a slightly larger proportion of solid PSCs (red-yellow sectors) in 2011 (63\%), 2012 (58\%), and $2014(57 \%)$.

\section{Conclusions}

A decade of MLS and CALIOP satellite measurements from 2006 to 2015 over the Antarctic polar vortex were used to investigate the early season development of PSCs and the gasphase $\mathrm{HNO}_{3}$ distribution in the lower stratosphere. We developed a compact visual representation of the daily orbit tracks that allows a time series to be constructed from the montage of a few hundred separate daily images, consisting of a combination of different days/pressure levels/species, and displayed on a single page.

Lidar properties were calculated for the STS and NAT components of PSCs. We reviewed the capabilities of spaceborne instruments (lidar, mid-infrared, microwave) applied to the detection of PSCs and uptake of $\mathrm{HNO}_{3}$ and used the results to investigate the generation of large particle/low number density NAT at temperatures above the ice frost point, which results in sub-visible PSCs that can be detected through the gas-phase uptake of $\mathrm{HNO}_{3}$. The presence of subvisible PSCs was found in over half of the years examined.
In this work we have demonstrated that the early initiation of NAT nucleation in the Antarctic vortex takes place frequently at temperatures above the ice frost point and often before cooling produces liquid PSCs. A consistent picture emerges, with $\mathrm{HNO}_{3}$ depletion occurring in the inner vortex, usually before any associated PSC development is detected by CALIOP. We conclude that an ice-seeding process is not essential for the initiation of NAT nucleation or subsequent development of large-scale NAT growth in the early winter.

We used detailed measurements in the CALIOP and MLS along-track transects to illustrate that the formation of PSCs is not only governed by the local ambient temperature, but is also shaped in large measure by the underlying temperature histories.

A cluster analysis method was used to organize the combined CALIOP and MLS data into a manageable form to guide an investigation of the decadal climatology and facilitate ready comparison of the patterns of interannual variability. The temperature distribution of the groups was used to compare the relative frequency of formation of liquid and solid PSCs in the Antarctic lower polar stratosphere.

\section{Data availability}

MLS data are archived at the NASA Goddard Earth Sciences Data Information and Services Center (Lambert et al., 2015; Manney et al., 2015).

CALIOP data were obtained from the NASA Langley Research Center Atmospheric Science Data Center (CALIPSO Science Team, 2015a, b).

GEOS5.9.1 data were obtained from the Goddard Earth Sciences Data and Information Services Center (GMAO, 2013).

MERRA-2 data were obtained from the Goddard Earth Sciences Data and Information Services Center (GMAO, 2015).

IDL software for calculation of PSC thermodynamic properties provided by M. E. Hervig was obtained from the GATS Scientific Software 95 website (http://gwest.gats-inc. com/software/software_page.html). Fortran software for Tmatrix calculations provided by M. I. Mishchenko was obtained from the NASA GISS website (http://www.giss.nasa. gov/staff/mmishchenko/t_matrix.html).

Acknowledgements. We gratefully acknowledge members of the teams associated with the CALIOP and MLS instruments, and the GEOS-5 meteorological analyses. Work at the Jet Propulsion Laboratory, California Institute of Technology, was carried out under a contract with the National Aeronautics and Space Administration. We acknowledge the scientific guidance and sponsorship of the World Climate Research Programme, coordinated in the framework of the SPARC (Stratosphere-troposphere Processes And their Role in Climate) Polar Stratospheric Clouds activity. We acknowledge the International Space Science Institute (ISSI), Bern, Switzerland, 
for their support of the Polar Stratospheric Cloud initiative. We thank the anonymous reviewers for their careful reading of the manuscript and their comments and suggestions.

Edited by: F. Khosrawi

Reviewed by: two anonymous referees

\section{References}

Biele, J., Tsias, A., Luo, B. P., Carslaw, K. S., Neuber, R., Beyerle, G., and Peter, T.: Nonequilibrium coexistence of solid and liquid particles in Arctic stratospheric clouds, J. Geophys. Res., 106, 22991-23007, 2001.

Bosilovich, M., Akella, S., Coy, L., Cullather, R., Draper, C., Gelaro, R., Kovach, R., Liu, Q., Molod, A., Norris, P., Wargan, K., Chao, W., Reichle, R., Takacs, L., Vikhliaev, Y., Bloom, S., Collow, A., Firth, S., Labow, G., Partyka, G., Pawson, S., Reale, O., Schubert, S. D., and Suarez, M.: MERRA-2: Initial Evaluation of the Climate, Tech. rep., NASA Goddard Space Flight Center, Greenbelt, MD, NASA Tech. Rep. Series on Global Modeling and Data Assimilation, NASA/TM-2015-104606, Vol. 43, 2015.

CALIPSO Science Team: CALIPSO/CALIOP Level 1B, Lidar Profile Data, version 3.01, Hampton, VA, USA: NASA Atmospheric Science Data Center (ASDC), doi:10.5067/CALIOP/CALIPSO/CAL_LID_L1-ValStage1V3-01_L1B-003.01, 2015a.

CALIPSO Science Team: CALIPSO/CALIOP Level 2, Polar Stratospheric Cloud Data, version 1.00, Hampton, VA, USA: NASA Atmospheric Science Data Center (ASDC), doi:10.5067/CALIOP/CALIPSO/CAL_LID_L2_PSCMaskProv-V1-00_L2-001.00, 2015b.

Carlsaw, K. S., Luo, B. P., Clegg, S. L., Peter, T., Brimblecombe, P., and Crutzen, P. J.: Stratospheric aerosol growth and $\mathrm{HNO}_{3}$ gas phase depletion from coupled $\mathrm{HNO}_{3}$ and water uptake by liquid particles, Geophys. Res. Lett., 21, 2479-2482, 1994.

Carlsaw, K. S., Luo, B. P., and Peter, T.: An analytic expression for the composition of aqueous $\mathrm{HNO}_{3}-\mathrm{H}_{2} \mathrm{SO}_{4}$ stratospheric aerosols including gas phase removal of $\mathrm{HNO}_{3}$, Geophys. Res. Lett., 22, 1877-1880, 1995.

Carslaw, K. S., Peter, T., and Clegg, S. L.: Modeling the composition of liquid stratospheric aerosols, Rev. Geophys., 35, 125-154, 1997.

Carslaw, K. S., Kettleborough, J. A., Northway, M. J., Davies, S., Gao, R.-S., Fahey, D. W., Baumgardner, D. G., Chipperfield, M. P., and Kleinböhl, A.: A vortex-scale simulation of the growth and sedimentation of large nitric acid hydrate particles, J. Geophys. Res., 107, 8300, doi:10.1029/2001JD000467, 2002.

Curtius, J., Weigel, R., Vössing, H.-J., Wernli, H., Werner, A., Volk, C.-M., Konopka, P., Krebsbach, M., Schiller, C., Roiger, A., Schlager, H., Dreiling, V., and Borrmann, S.: Observations of meteoric material and implications for aerosol nucleation in the winter Arctic lower stratosphere derived from in situ particle measurements, Atmos. Chem. Phys., 5, 3053-3069, doi:10.5194/acp-5-3053-2005, 2005.

Drdla, K., Gandrud, B. W., Baumgardner, D., Wilson, J. C., Bui, T. P., Hurst, D., Schauffler, S. M., Jost, H., Greenblatt, J. B., and Webster, C. R.: Evidence for the widespread presence of liquid- phase particles during the 1999-2000 Arctic winter, J. Geophys. Res., 108, 8318, doi:10.1029/2001JD001127, 2003.

Engel, I., Luo, B. P., Pitts, M. C., Poole, L. R., Hoyle, C. R., Grooß, J.-U., Dörnbrack, A., and Peter, T.: Heterogeneous formation of polar stratospheric clouds - Part 2: Nucleation of ice on synoptic scales, Atmos. Chem. Phys., 13, 10769-10785, doi:10.5194/acp13-10769-2013, 2013.

Flentje, H., Dörnbrack, A., Fix, A., Meister, A., Schmid, H., Füglistaler, S., Luo, B., and Peter, T.: Denitrification inside the stratospheric vortex in the winter of 1999-2000 by sedimentation of large nitric acid trihydrate particles, J. Geophys. Res., 107, AAC 11-1-AAC 11-15, doi:10.1029/2001JD001015, 2002.

GMAO (Global Modeling and Assimilation Office): GEOS5.9.1 v1.2 NRT Assimilation Products FP-IT (Forward Processing for Instrument Teams), available by subscription at: https:// gmao.gsfc.nasa.gov/products/index.php (last access: 2 December 2016), 2013.

GMAO (Global Modeling and Assimilation Office): MERRA2 inst3_3d_asm_Nv: 3d,3-Hourly,Instantaneous,ModelLevel,Assimilation,Assimilated Meteorological Fields V5.12.4, Greenbelt, MD, USA, Goddard Earth Sciences Data and Information Services Center (GES DISC), doi:10.5067/WWQSXQ8IVFW8, 2015.

Grooß, J.-U., Engel, I., Borrmann, S., Frey, W., Günther, G., Hoyle, C. R., Kivi, R., Luo, B. P., Molleker, S., Peter, T., Pitts, M. C., Schlager, H., Stiller, G., Vömel, H., Walker, K. A., and Müller, R.: Nitric acid trihydrate nucleation and denitrification in the Arctic stratosphere, Atmos. Chem. Phys., 14, 1055-1073, doi:10.5194/acp-14-1055-2014, 2014.

Hanson, D. and Mauersberger, K.: Laboratory studies of the nitric acid trihydrate: Implications for the south polar stratosphere, Geophys. Res. Lett., 15, 855-858, 1988.

Höpfner, M.: Study on the impact of polar stratospheric clouds on high resolution mid-IR limb emission spectra, J. Quant. Spectrosc. Ra., 83, 93-107, doi:10.1016/S0022-4073(02)002996, 2004.

Höpfner, M., Larsen, N., Spang, R., Luo, B. P., Ma, J., Svendsen, S. H., Eckermann, S. D., Knudsen, B., Massoli, P., Cairo, F., Stiller, G., v. Clarmann, T., and Fischer, H.: MIPAS detects Antarctic stratospheric belt of NAT PSCs caused by mountain waves, Atmos. Chem. Phys., 6, 1221-1230, doi:10.5194/acp-6-1221-2006, 2006a.

Höpfner, M., Luo, B. P., Massoli, P., Cairo, F., Spang, R., Snels, M., Di Donfrancesco, G., Stiller, G., von Clarmann, T., Fischer, H., and Biermann, U.: Spectroscopic evidence for NAT, STS, and ice in MIPAS infrared limb emission measurements of polar stratospheric clouds, Atmos. Chem. Phys., 6, 1201-1219, doi:10.5194/acp-6-1201-2006, 2006b.

Höpfner, M., Pitts, M. C., and Poole, L. R.: Comparison between CALIPSO and MIPAS observations of polar stratospheric clouds, J. Geophys. Res., 114, D00H05, doi:10.1029/2009JD012114, 2009.

Hoyle, C. R., Engel, I., Luo, B. P., Pitts, M. C., Poole, L. R., Grooß, J.-U., and Peter, T.: Heterogeneous formation of polar stratospheric clouds - Part 1: Nucleation of nitric acid trihydrate (NAT), Atmos. Chem. Phys., 13, 9577-9595, doi:10.5194/acp13-9577-2013, 2013. 
Jain, A. K.: Data clustering: 50 years beyond K-means, Pattern Recogn. Lett., 31, 651-666, doi:10.1016/j.patrec.2009.09.011, 2010.

Jensen, E. J., Toon, O. B., Tabazadeh, A., and Drdla, K.: Impact of polar stratospheric cloud particle composition, number density, and lifetime on denitrification, J. Geophys. Res., 107, 8284, doi:10.1029/2001JD000440, 2002.

Koop, T., Biermann, U. M., Raber, W., Luo, B. P., Crutzen, P. J., and Peter, T.: Do stratospheric aerosol droplets freeze above the ice frost point?, Geophys. Res. Lett., 22, 917-920, 1995.

Lambert, A., Grainger, R. G., Remedios, J. J., Reburn, W. J., Rodgers, C. D., Taylor, F. W., Roche, A. E., Kumer, J. B., Massie, S. T., and Deshler, T.: Validation of aerosol measurements from the improved stratospheric and mesospheric sounder, J. Geophys. Res., 101, 9811-9830, doi:10.1029/95JD01702, 1996.

Lambert, A., Read, W. G., Livesey, N. J., Santee, M. L., Manney, G. L., Froidevaux, L., Wu, D. L., Schwartz, M. J., Pumphrey, H. C., Jimenez, C., Nedoluha, G. E., Cofield, R. E., Cuddy, D. T., Daffer, W. H., Drouin, B. J., Fuller, R. A., Jarnot, R. F., Knosp, B. W., Pickett, H. M., Perun, V. S., Snyder, W. V., Stek, P. C., Thurstans, R. P., Wagner, P. A., Waters, J. W., Jucks, K. W., Toon, G. C., Stachnik, R. A., Bernath, P. F., Boone, C. D., Walker, K. A., Urban, J., Murtagh, D., Elkins, J. W., and Atlas, E.: Validation of the Aura Microwave Limb Sounder middle atmosphere water vapor and nitrous oxide measurements, J. Geophys. Res., 112, D24S36, doi:10.1029/2007JD008724, 2007.

Lambert, A., Santee, M. L., Wu, D. L., and Chae, J. H.: A-train CALIOP and MLS observations of early winter Antarctic polar stratospheric clouds and nitric acid in 2008, Atmos. Chem. Phys., 12, 2899-2931, doi:10.5194/acp-12-2899-2012, 2012.

Lambert, A., Read, W., and Livesey, N.: MLS/Aura Level 2 Water Vapor $\left(\mathrm{H}_{2} \mathrm{O}\right)$ Mixing Ratio V004, Greenbelt, MD, USA, Goddard Earth Sciences Data and Information Services Center (GES DISC), doi:10.5067/AURA/MLS/DATA2009, 2015.

Lawrence, Z. D., Manney, G. L., Minschwaner, K., Santee, M. L., and Lambert, A.: Comparisons of polar processing diagnostics from 34 years of the ERA-Interim and MERRA reanalyses, Atmos. Chem. Phys., 15, 3873-3892, doi:10.5194/acp-15-38732015, 2015.

Liu, L. and Mishchenko, M. I.: Constraints on PSC particle microphysics derived from lidar observations, J. Quant. Spectrosc. Ra., 70, 817-831, doi:10.1016/S0022-4073(01)00048-6, 2001.

Livesey, N. J., Snyder, W. V., Read, W. G., and Wagner, P. A.: Retrieval algorithms for the EOS Microwave Limb Sounder (MLS), IEEE T. Geosci. Remote Sens., 44, 1144-1155, 2006.

Livesey, N. J., Santee, M. L., and Manney, G. L.: A Match-based approach to the estimation of polar stratospheric ozone loss using Aura Microwave Limb Sounder observations, Atmos. Chem. Phys., 15, 9945-9963, doi:10.5194/acp-15-9945-2015, 2015.

Livesey, N. J., Read, W. G., Wagner, P. A., Froidevaux, L., Lambert, A., Manney, G. L., Valle, L. F. M., Pumphrey, H. C., Santee, M. L., Schwartz, M. J., Wang, S., Fuller, R. A., Jarnot, R. F., Knosp, B. W., and Martinez, E.: Version 4.2x Level 2 data quality and description document, Tech. Rep. JPL D-33509, Jet Propulsion Laboratory, available at: http://mls.jpl.nasa.gov (last access: 2 December 2016), 2016.

Luo, B., Voigt, C., Fueglistaler, S., and Peter, T.: Extreme NAT supersaturations in mountain wave ice PSCs: A clue to NAT forma- tion, J. Geophys. Res., 108, 4441, doi:10.1029/2002JD003104, 2003.

Manney, G. L., Zurek, R. W., O’Neill, A., and Swinbank, R.: On the motion of air through the stratospheric polar vortex, J. Atmos. Sci., 51, 2973-2994, 1994.

Manney, G. L., Daffer, W. H., Zawodny, J. M., Bernath, P. F., Hoppel, K. W., Walker, K. A., Knosp, B. W., Boone, C., Remsberg, E. E., Santee, M. L., Harvey, V. L., Pawson, S., Jackson, D. R., Deaver, L., McElroy, C. T., McLinden, C. A., Drummond, J. R., Pumphrey, H. C., Lambert, A., Schwartz, M. J., Froidevaux, L., McLeod, S., Takacs, L. L., Suarez, M. J., Trepte, C. R., Cuddy, D. C., Livesey, N. J., Harwood, R. S., and Waters, J. W.: Solar occultation satellite data and derived meteorological products: Sampling issues and comparisons with Aura MLS, J. Geophys. Res., 112, D24S50, doi:10.1029/2007JD008709, 2007.

Manney, G., Santee, M., Froidevaux, L., Livesey, N., and Read, W.: MLS/Aura Level 2 Nitric Acid $\left(\mathrm{HNO}_{3}\right)$ Mixing Ratio V004, Greenbelt, MD, USA, Goddard Earth Sciences Data and Information Services Center (GES DISC), doi:10.5067/AURA/MLS/DATA2012, 2015.

Massie, S., Gille, J., Khosravi, R., Lee, H., Kinnison, D., Francis, G., Nardi, B., Eden, T., Craig, C., Halvorson, C., Coffey, M., Packman, D., Cavanaugh, C., Craft, J., Dean, V., Ellis, D., Barnett, J., Hepplewhite, C., Lambert, A., Manney, G., Strawa, A., and Legg, M.: High Resolution Dynamics Limb Sounder observations of polar stratospheric clouds and subvisible cirrus, J. Geophys. Res., 112, D24S31, doi:10.1029/2007JD008788, 2007.

Mishchenko, M. I. and Travis, L. D.: Capabilities and limitations of a current FORTRAN implementation of the T-matrix method for randomly oriented, rotationally symmetric scatterers, J. Quant. Spectrosc. Ra., 60, 309-324, doi:10.1016/S00224073(98)00008-9, 1998.

Molleker, S., Borrmann, S., Schlager, H., Luo, B., Frey, W., Klingebiel, M., Weigel, R., Ebert, M., Mitev, V., Matthey, R., Woiwode, W., Oelhaf, H., Dörnbrack, A., Stratmann, G., Grooß, J.U., Günther, G., Vogel, B., Müller, R., Krämer, M., Meyer, J., and Cairo, F.: Microphysical properties of synoptic-scale polar stratospheric clouds: in situ measurements of unexpectedly large $\mathrm{HNO}_{3}$-containing particles in the Arctic vortex, Atmos. Chem. Phys., 14, 10785-10801, doi:10.5194/acp-14-10785-2014, 2014.

Murphy, D. M. and Koop, T.: Review of the vapour pressures of ice and supercooled water for atmospheric applications, Q. J. Roy. Meteor. Soc., 131, 1539-1565, 2005.

Myhre, C. E. L., Grothe, H., Gola, A. A., and Nielsen, C. J.: Optical Constants of $\mathrm{HNO}_{3} / \mathrm{H}_{2} \mathrm{O}$ and $\mathrm{H}_{2} \mathrm{SO}_{4} / \mathrm{HNO}_{3} / \mathrm{H}_{2} \mathrm{O}$ at Low Temperatures in the Infrared Region, J. Phys. Chem. A, 109, 71667171, doi:10.1021/jp0508406, 2005.

Nousiainen, T., Zubko, E., Lindqvist, H., Kahnert, M., and Tyynela, J.: Comparison of scattering by different nonspherical, wavelength-scale particles, J. Quant. Spectrosc. Ra., 113, 121135, doi:10.1016/j.jqsrt.2012.03.032, 2012.

Pitts, M. C., Poole, L. R., and Thomason, L. W.: CALIPSO polar stratospheric cloud observations: second-generation detection algorithm and composition discrimination, Atmos. Chem. Phys., 9, 7577-7589, doi:10.5194/acp-9-7577-2009, 2009.

Pitts, M. C., Poole, L. R., Lambert, A., and Thomason, L. W.: An assessment of CALIOP polar stratospheric cloud composition classification, Atmos. Chem. Phys., 13, 2975-2988, doi:10.5194/acp-13-2975-2013, 2013. 
Read, W. G., Lambert, A., Bacmeister, J., Cofield, R. E., Christensen, L. E., Cuddy, D. T., Daffer, W. H., Drouin, B. J., Fetzer, E., Froidevaux, L., Fuller, R., Herman, R., Jarnot, R. F., Jiang, J. H., Jiang, Y. B., Kelly, K., Knosp, B. W., Kovalenko, L. J., Livesey, N. J., Liu, H. C., Manney, G. L., Pickett, H. M., Pumphrey, H. C., Rosenlof, K. H., Sabounchi, X., Santee, M. L., Schwartz, M. J., Snyder, W. V., Stek, P. C., Su, H., Takacs, L. L., Thurstans, R. P., Vomel, H., Wagner, P. A., Waters, J. W., Webster, C. R., Weinstock, E. M., and Wu, D. L.: Aura Microwave Limb Sounder upper tropospheric and lower stratospheric $\mathrm{H}_{2} \mathrm{O}$ and relative humidity with respect to ice validation, J. Geophys. Res., 112, D24S35, doi:10.1029/2007JD008752, 2007.

Rienecker, M. M., Suarez, M. J., Todling, R., Bacmeister, K., Takacs, L., Liu, H.-C., Gu, W., Sienkiewicz, M., Koster, R. D., Gelaro, R., Stajner, I., and Nielsen, J. E.: The GEOS-5 Data Assimilation System - Documentation of Versions 5.0.1, 5.1.0, and 5.2.0, Tech. rep., NASA Goddard Space Flight Center, Greenbelt, MD, NASA/TM-2008-10406, Vol. 27, 2008.

Santee, M. L., Lambert, A., Read, W. G., Livesey, N. J., Cofield, R. E., Cuddy, D. T., Daffer, W. H., Drouin, B. J., Froidevaux, L., Fuller, R. A., Jarnot, R. F., Knosp, B. W., Manney, G. L., Perun, V. S., Snyder, W. V., Stek, P. C., Thurstans, R. P., Wagner, P. A., Waters, J. W., Muscari, G., de Zafra, R. L., Dibb, J. E., Fahey, D. W., Popp, P. J., Marcy, T. P., Jucks, K. W., Toon, G. C., Stachnik, R. A., Bernath, P. F., Boone, C. D., Walker, K. A., Urban, J., and Murtagh, D.: Validation of the Aura Microwave Limb Sounder $\mathrm{HNO}_{3}$ measurements, J. Geophys. Res., 112, D24S40, doi:10.1029/2007JD008721, 2007.

Spang, R. and Remedios, J. J.: Observations of a distinctive infrared spectral feature in the atmospheric spectra of polar stratospheric clouds measured by the CRISTA instrument, Geophys. Res. Lett., 30, 1875, doi:10.1029/2003GL017231, 2003.

Spang, R., Hoffmann, L., Höpfner, M., Griessbach, S., Müller, R., Pitts, M. C., Orr, A. M. W., and Riese, M.: A multi-wavelength classification method for polar stratospheric cloud types using infrared limb spectra, Atmos. Meas. Tech., 9, 3619-3639, doi:10.5194/amt-9-3619-2016, 2016.

Tabazadeh, A., Jensen, E. J., Toon, O. B., Drdla, K., and Schoeberl, M. R.: Role of the stratospheric polar freezing belt in denitrification, Science, 291, 2591-2594, 2001.

Tabazadeh, A., Djikaev, Y. S., Hamill, P., and Reiss, H.: Laboratory evidence for surface nucleation of solid polar stratospheric cloud particles, J. Phys. Chem. A, 106, 10238-10246, 2002.

Tibshirani, R., Walther, G., and Hastie, T.: Estimating the number of clusters in a data set via the gap statistic, J. Roy. Stat. Soc. B, 63, 411-423, doi:10.1111/1467-9868.00293, 2001.

Toon, O. B., Turco, R. P., Jordan, J., Goodman, J., and Ferry, G.: Physical processes in polar stratospheric ice clouds, J. Geophys. Res., 94, 11359-11380, 1989.

Toon, O. B., Tolbert, M. A., Koehler, B. G., Middlebrook, A. M., and Jordan, J.: Infrared optical constants of $\mathrm{H}_{2} \mathrm{O}$-ice, amorphous acid solutions, and nitric acid hydrates, J. Geophys. Res., 99, 25631-25654, 1994.

Turco, R. P., Toon, O. B., and Hamill, P.: Heterogeneous physicochemistry of the polar ozone hole, J. Geophys. Res., 94, 16493$16510,1989$.

Voigt, C., Schlager, H., Luo, B. P., Dörnbrack, A., Roiger, A., Stock, P., Curtius, J., Vössing, H., Borrmann, S., Davies, S., Konopka, P., Schiller, C., Shur, G., and Peter, T.: Nitric Acid
Trihydrate (NAT) formation at low NAT supersaturation in Polar Stratospheric Clouds (PSCs), Atmos. Chem. Phys., 5, 13711380, doi:10.5194/acp-5-1371-2005, 2005.

von Hobe, M., Bekki, S., Borrmann, S., Cairo, F., D’Amato, F., Di Donfrancesco, G., Dörnbrack, A., Ebersoldt, A., Ebert, M., Emde, C., Engel, I., Ern, M., Frey, W., Genco, S., Griessbach, S., Grooß, J.-U., Gulde, T., Günther, G., Hösen, E., Hoffmann, L., Homonnai, V., Hoyle, C. R., Isaksen, I. S. A., Jackson, D. R., Jánosi, I. M., Jones, R. L., Kandler, K., Kalicinsky, C., Keil, A., Khaykin, S. M., Khosrawi, F., Kivi, R., Kuttippurath, J., Laube, J. C., Lefèvre, F., Lehmann, R., Ludmann, S., Luo, B. P., Marchand, M., Meyer, J., Mitev, V., Molleker, S., Müller, R., Oelhaf, H., Olschewski, F., Orsolini, Y., Peter, T., Pfeilsticker, K., Piesch, C., Pitts, M. C., Poole, L. R., Pope, F. D., Ravegnani, F., Rex, M., Riese, M., Röckmann, T., Rognerud, B., Roiger, A., Rolf, C., Santee, M. L., Scheibe, M., Schiller, C., Schlager, H., Siciliani de Cumis, M., Sitnikov, N., Søvde, O. A., Spang, R., Spelten, N., Stordal, F., Suminska-Ebersoldt, O., Ulanovski, A., Ungermann, J., Viciani, S., Volk, C. M., vom Scheidt, M., von der Gathen, P., Walker, K., Wegner, T., Weigel, R., Weinbruch, S., Wetzel, G., Wienhold, F. G., Wohltmann, I., Woiwode, W., Young, I. A. K., Yushkov, V., Zobrist, B., and Stroh, F.: Reconciliation of essential process parameters for an enhanced predictability of Arctic stratospheric ozone loss and its climate interactions (RECONCILE): activities and results, Atmos. Chem. Phys., 13, 92339268, doi:10.5194/acp-13-9233-2013, 2013.

Ward, S. M., Deshler, T., and Hertzog, A.: Quasi-Lagrangian measurements of nitric acid trihydrate formation over Antarctica, J. Geophys. Res.-Atmos., 119, 245-258, doi:10.1002/2013JD020326, 2014.

Waters, J. W., Froidevaux, L., Harwood, R. S., Jarnot, R. F., Pickett, H. M., Read, W. G., Siegel, P. H., Cofield, R. E., Filipiak, M. J., Flower, D. A., Holden, J. R., Lau, G. K. K., Livesey, N. J., Manney, G. L., Pumphrey, H. C., Santee, M. L., Wu, D. L., Cuddy, D. T., Lay, R. R., Loo, M. S., Perun, V. S., Schwartz, M. J., Stek, P. C., Thurstans, R. P., Boyles, M. A., Chandra, K. M., Chavez, M. C., Chen, G. S., Chudasama, B. V., Dodge, R., Fuller, R. A., Girard, M. A., Jiang, J. H., Jiang, Y. B., Knosp, B. W., LaBelle, R. C., Lam, J. C., Lee, K. A., Miller, D., Oswald, J. E., Patel, N. C., Pukala, D. M., Quintero, O., Scaff, D., Van Snyder, W., Tope, M. C., Wagner, P. A., and Walch, M. J.: The Earth Observing System Microwave Limb Sounder (EOS MLS) on the Aura satellite, IEEE T. Geosci. Remote Sens., 44, 1075-1092, 2006.

Winker, D. M., Vaughan, M. A., Omar, A., Hu, Y., Powell, K. A., Liu, Z., Hunt, W. H., and Young, S. A.: Overview of the CALIPSO Mission and CALIOP data processing algorithms, J. Atmos. Ocean. Tech., 26, 2310-2323, doi:10.1175/2009JTECHA1281.1, 2009.

Woiwode, W., Grooß, J.-U., Oelhaf, H., Molleker, S., Borrmann, S., Ebersoldt, A., Frey, W., Gulde, T., Khaykin, S., Maucher, G., Piesch, C., and Orphal, J.: Denitrification by large NAT particles: the impact of reduced settling velocities and hints on particle characteristics, Atmos. Chem. Phys., 14, 11525-11544, doi:10.5194/acp-14-11525-2014, 2014.

Woiwode, W., Höpfner, M., Bi, L., Pitts, M. C., Poole, L. R., Oelhaf, H., Molleker, S., Borrmann, S., Klingebiel, M., Belyaev, G., Ebersoldt, A., Griessbach, S., Grooß, J.-U., Gulde, T., Krämer, M., Maucher, G., Piesch, C., Rolf, C., Sartorius, C., Spang, R., and Orphal, J.: Spectroscopic evidence of large aspheri- 
cal $\beta$-NAT particles involved in denitrification in the December 2011 Arctic stratosphere, Atmos. Chem. Phys., 16, 9505-9532, doi:10.5194/acp-16-9505-2016, 2016.

Zhu, Y., Toon, O. B., Lambert, A., Kinnison, D. E., Brakebusch, M., Bardeen, C. G., Mills, M. J., and English, J. M.: Development of a Polar Stratospheric Cloud Model within the Community Earth System Model using constraints on Type I PSCs from the 2010-2011 Arctic winter, J. Adv. Model. Earth Syst., 7, 551585, doi:10.1002/2015MS000427, 2015.
Zhu, Y., Toon, O. B., Pitts, M. C., Lambert, A., Bardeen, C., and Kinnison, D. E.: Comparing simulated PSC optical properties with CALIPSO observations during the 2010 Antarctic Winter, J. Geophys. Res., in review, 2016. 